\title{
MoGLN2 Is Important for Vegetative Growth, Conidiogenesis, Maintenance of Cell Wall Integrity and Pathogenesis of Magnaporthe oryzae
}

\author{
Osakina Aron ${ }^{1,2}$, Min Wang ${ }^{2}$, Lianyu Lin ${ }^{1}$, Wajjiha Batool ${ }^{1,2}$, Birong Lin ${ }^{2}$, Ammarah Shabbir ${ }^{1}$, \\ Zonghua Wang $1,2,3, *(D)$ and Wei Tang ${ }^{1,2, *}$ \\ 1 Fujian Universities Key Laboratory for Plant-Microbe Interaction, College of Plant Protection, Fujian \\ Agriculture and Forestry University, Fuzhou 350002, China; osakina.aron@yahoo.com (O.A.); \\ lianyully@163.com (L.L.); jiaali174@yahoo.com (W.B.); ammara.shabbir@yahoo.com (A.S.) \\ 2 State Key Laboratory of Ecological Pest Control for Fujian and Taiwan Crops, Fujian Agriculture and Forestry \\ University, Fuzhou 350002, China; 17750243431@139.com (M.W.); lin18105028780@163.com (B.L.) \\ 3 Marine and Agricultural Biotechnology Center, Institute of Oceanography, Minjiang University, \\ Fuzhou 350108, China \\ * Correspondence: wangzh@fafu.edu.cn (Z.W.); tangw@fafu.edu.cn (W.T.)
}

check for updates

Citation: Aron, O.; Wang, M.; Lin, L. Batool, W.; Lin, B.; Shabbir, A.; Wang, Z.; Tang, W. MoGLN2 Is Important for Vegetative Growth, Conidiogenesis, Maintenance of Cell Wall Integrity and Pathogenesis of Magnaporthe oryzae. J. Fungi 2021, 7, 463. https://doi.org/10.3390/jof7060463

Academic Editor: Raffaella Maria Balestrini

Received: 7 May 2021

Accepted: 6 June 2021

Published: 8 June 2021

Publisher's Note: MDPI stays neutral with regard to jurisdictional claims in published maps and institutional affiliations.

Copyright: (c) 2021 by the authors. Licensee MDPI, Basel, Switzerland. This article is an open access article distributed under the terms and conditions of the Creative Commons Attribution (CC BY) license (https:// creativecommons.org/licenses/by/ $4.0 /)$

\begin{abstract}
Glutamine is a non-essential amino acid that acts as a principal source of nitrogen and nucleic acid biosynthesis in living organisms. In Saccharomyces cerevisiae, glutamine synthetase catalyzes the synthesis of glutamine. To determine the role of glutamine synthetase in the development and pathogenicity of plant fungal pathogens, we used S. cerevisiae Gln1 amino acid sequence to identify its orthologs in Magnaporthe oryzae and named them MoGln1, MoGln2, and MoGln3. Deletion of MoGLN1 and MoGLN3 showed that they are not involved in the development and pathogenesis of $M$. oryzae. Conversely, $\Delta M o g \ln 2$ was reduced in vegetative growth, experienced attenuated growth on Minimal Medium (MM), and exhibited hyphal autolysis on oatmeal and straw decoction and corn media. Exogenous L-glutamine rescued the growth of $\Delta M o g \ln 2$ on MM. The $\Delta M o g \ln 2$ mutant failed to produce spores and was nonpathogenic on barley leaves, as it was unable to form an appressoriumlike structure from its hyphal tips. Furthermore, deletion of MoGLN2 altered the fungal cell wall integrity, with the $\Delta M o g \ln 2$ mutant being hypersensitive to $\mathrm{H}_{2} \mathrm{O}_{2}$. MoGln1, MoGln2, and MoGln3 are located in the cytoplasm. Taken together, our results shows that MoGLN2 is important for vegetative growth, conidiation, appressorium formation, maintenance of cell wall integrity, oxidative stress tolerance and pathogenesis of M. oryzae.
\end{abstract}

Keywords: glutamine; pathogenicity; glutamine synthetase; Magnaporthe oryzae; cell wall integrity

\section{Introduction}

Glutamine is a non-essential amino acid and is required in a vast number of metabolic pathways in living organisms. For example, in humans glutamine is required in pathways such as nitrogen metabolism, ammonia detoxification, acid-base homeostasis, osmotic regulation, cell signaling, and proliferation [1,2]. In addition, it has been reported that glutamine acts as a precursor for neurotransmitters and a substrate for immune cells $[3,4]$. Furthermore, it has been shown that glutamine is used for the synthesis of biomolecules such as glucose, purines, pyrimidines, adenosine monophosphate, and nicotinamide adenine dinucleotide (NAD+) [5-7]. Since glutamine is a crucial metabolite in the metabolism of nitrogen, the intracellular glutamine levels are tightly regulated. Experimental data for various fungi have provided evidence that glutamine is a key effector of nitrogen catabolite repression (NCR), a regulatory cascade that is biased toward or prefers the utilization of reduced nitrogen sources such as ammonium and glutamine at the expense of more complex and energy-demanding ones, e.g., nitrate, purines, and proteins [8,9]. A classic example occurs in Saccharomyces cerevisiae, where a well-established model of 
the target of rapamycin (Tor) exists, where intracellular glutamine levels are sensed by the (Tor) complex kinase 1 (TorC1), thus relaying the signal of glutamine availability to the GATA-type transcription factors Gln3 and Gat1. These GATA factors then respond by activating the transcription of NCR-sensitive genes during nitrogen-starvation conditions or when non-preferred nitrogen sources are present $[10,11]$. Glutamine signals for nitrogen metabolite repression can also be sensed from ammonia, glutamate, and nitrate [12-14].

Glutamine synthetase (GS) catalyzes the biosynthesis of glutamine, which acts as a principal nitrogen source for the synthesis of nucleic acid and protein synthesis. In living cells, ammonium assimilation occurs in two main ways [15]: NADP-dependent glutamate synthesis, a reaction catalyzed by glutamate dehydrogenases, in which ammonium and 2oxoglutarate act as the substrates [16]; and the assimilation of ammonium by the glutamine synthetase, an ATP-dependent reaction that results into the formation of an intermediate product $\gamma$-glutamyl phosphate from glutamate, which eventually combines with ammonia to generate glutamine and inorganic phosphate $[15,16]$. Several researchers have studied the biological role, physico-chemical properties, and kinetic properties of GS from different sources [17]. Methionine sulfoximine (MetSox) and phosphinothricin (PPT) have been reported to be the inhibitors of GS activity, as they tightly bind to its active site of GS [16]. Because of the inhibition property of these two molecules on GS, GS can act as an important target for bio-pesticides to be used in the agricultural industry. GS exhibits both the biosynthetic and $\gamma$-glutamyl transferase activities, with these two different forms based on whether the GS is in adenylylated or non-adenylylated forms $[18,19]$. The biosynthetic activity catalyzes the formation of glutamine from glutamate and ammonia, whereas the $\gamma$-glutamyl transfer activity catalyzes the transfer of $\gamma$-glutamyl moieties to peptides, amino acids, or water [20]. GS is classified as GSI, GSII, or GSIII [21]. GSI enzymes were thought to exist exclusively in prokaryotes, and their structures were shown to be dodecameric [22,23]. Later, GSI enzymes were also identified in mammals and plants [24,25]. In filamentous fungi, GSII family proteins were identified, and in most cases, as one GS-encoding gene present in the fungal genomes. However, two different subunits of GS $\alpha$ and GS $\beta$ were found to encode the GSII family in the filamentous fungus Neurospora crassa [26,27]. The presence of these two GS encoding genes in N. crassa was confirmed by genome sequencing. Since GS plays a crucial role in glutamine biosynthesis and nitrogen regulation. The activity of GS is tightly regulated to a level that allows the amount of glutamine available for various metabolic pathways to be strictly controlled. Studies on how GS is regulated have clearly been shown in Prokaryotes. For instance, in Escherichia coli, glutamine and end products of glutamine metabolism, such as AMP, ADP, and other nucleotides, offer a cumulative feedback inhibition to GS1 by competing with the substrate glutamate for the active site [28]. In Bacillus subtilis, GSI activity is feedback-inhibited by glutamine [29] and this inhibited form of GS controls the DNA-binding capabilities of the TnrA and GlnR transcription factors via protein interaction [29,30]. Both these transcription factors eventually regulate gene expression in response to changes in nitrogen availability.

Because glutamine is the major substrate for numerous metabolic pathways, it is an important amino acid for normal functioning of living organisms; therefore, deficiency of glutamine caused by a defect in GS interferes with normal life. In Drosophila melanogaster, mutations in the gene encoding the mitochondrial glutamine synthetase I (GSI) resulted in embryo lethality, consequently leading to female sterility [31], Moreover, in mice, GS was also found to be essential in early embryogenesis [31]. In humans, mutations in the GLUL gene (OMIM: 138290), which encodes for GS, were reported to cause an ultra-rare recessive inborn error of metabolism - congenital glutamine synthetase deficiency [32], whereas in plants GS has been reported to be essential for normal plant growth and development [33]. In filamentous fungi such Aspergillus nidulans and Schizosaccharomyces pombe, GS plays a crucial role in growth and development [34,35], while deletion of the GS in plant pathogenic fungi Gibberella fujikuroi GS gene showed that, besides influencing growth, GS has a significant impact on the transcriptional control of primary and secondary metabolism [36]. 
Magnaporthe oryzae is a devastating hemibiotrophic fungus that attacks most cereals, including rice, thus posing a great challenge to global food security. Due to its economic significance and genetic tractability, the blast fungus has been developed as a model organism for plant-fungus interaction studies [37]. Infections begin when conidia germinate and develop a specialized dome-shaped structure called an appressorium upon coming in contact with the rice plant surface [38]. The mature appressorium then accumulates enormous turgor pressure $(8 \mathrm{MPa})$, which helps it puncture the rice cuticle, thus facilitating its entry into plant cells [39]. While inside the host cell, the fungus differentiates into bulbous invasive hyphae (IH), which eventually colonizes the adjacent plant cells. Rice blast fungus initially adopts a hemibiotrophic infection strategy, which lasts approximately four to six days; the fungus colonizes the living host cells without causing damage to the host at this stage. Later it enters into a devastating necrotrophic phase, where the fungus rapidly destroys the infected host tissue [40].

Previously, findings have documented the role of GS in growth and development in different organisms $[33,35,41]$. However, the exact influence of GS in the development and pathogenicity of plant fungal pathogens is unclear. In this study, we identified three genes that encode GS in rice blast fungus. We established that MoGLN1 and MoGLN3 had no influence on the development and pathogenicity of $M$. oryzae. In contrast, our findings showed that MoGLN2 is important in glutamine biosynthesis, exerting a significant effect on vegetative growth, conidiogenesis, appressorium-like structure formation, and melanin biosynthesis, and that it was crucial for the maintenance of cell wall integrity and oxidative stress tolerance in $M$. oryzae. Our findings, therefore, suggest that glutamine biosynthesis mediated by MoGLN2 could provide a suitable target point for antifungal design against plant fungal pathogens.

\section{Materials and Methods}

\subsection{Fungal Strains and Culture Conditions}

The wild-type (WT) Guy11 and mutant strains of M. oryzae were cultured at $25{ }^{\circ} \mathrm{C}$ using complete media (CM: $0.6 \%$ yeast extract, $0.6 \%$ casein hydrolysate, $1 \%$ sucrose, $1.5 \%$ agar) as described in [42]. Other media used in this study include minimal media (MM: $6 \mathrm{~g}$ of $\mathrm{NaNO}_{3}, 0.52 \mathrm{~g}$ of $\mathrm{KCl}, 0.52 \mathrm{~g}$ of $\mathrm{MgSO}_{4}, 1.52 \mathrm{~g}$ of $\mathrm{KH}_{2} \mathrm{PO}_{4}, 10 \mathrm{~g}$ of glucose, and $15 \mathrm{~g}$ of agar in $1 \mathrm{~L}$ of double-distilled water), straw decoction and corn media (SDC: $100 \mathrm{~g}$ of rice straw, $40 \mathrm{~g}$ of corn flour, and $15 \mathrm{~g}$ of agar in $1 \mathrm{~L}$ of double-distilled water), and oatmeal agar media (OTM: $50 \mathrm{~g}$ of oatmeal and $15 \mathrm{~g}$ of agar in $1 \mathrm{~L}$ of double-distilled water). Samples for genomic DNA extraction, total RNA, and protoplast preparation were cultured in liquid CM in an orbital shaker at $110 \mathrm{rpm}$ for 3 days.

Sensitivity assays were performed by culturing strains on CM plates supplemented with cell wall enforcing agents $(0.01 \%$ SDS, $200 \mu \mathrm{g} / \mathrm{mL}$ Congo red, $200 \mu \mathrm{g} / \mathrm{mL}$ Calcofluor white), oxidative stress agent $2.5 \mathrm{mM}$, and $5 \mathrm{mM}$ hydrogen peroxide $\left(\mathrm{H}_{2} \mathrm{O}_{2}\right)$ for 8 days at $28^{\circ} \mathrm{C}$ inside a dark chamber.

To induce conidiation, strains were cultured on rice bran agar ( $2 \%$ rice bran, $1.5 \%$ agar; $\mathrm{pH}$ 6.5) for 10 days at $28^{\circ} \mathrm{C}$ in the dark followed by 3 days of continuous light illumination. Conidia were collected in $5 \mathrm{~mL}$ of distilled water, filtered using three-layer lens paper, and counted with a hemocytometer under a light microscope.

\subsection{Target Gene Deletion and Complementation in M. oryzae}

To generate MoGLN deletion mutants, the split-marker approach [43] was adopted in the construction knockout vectors used for deleting each of the MoGLN genes in $M$. oryzae. The upstream and downstream flanking fragments of MoGLN genes were amplified with the primer pairs listed in Table S1. The amplified PCR fragments were ligated with hygromycin phosphotransferase (hph) cassette fragments amplified with primers HYG/F + HY/R and YG/R + HYG/R (Table S1) by overlapping PCR.

Protoplast preparation and transformation procedures were performed as previously described [44] Transformants were selected on TB3 medium supplemented $250 \mu \mathrm{g} / \mathrm{mL}$ hy- 
gromycin B (Roche Applied Science, Penzberg, Germany) and 200 нg/mL G418 (Invitrogen, Carlsbad, CA, USA), and the mutants were verified by Southern blotting analysis.

To generate complementation strains, fragments containing the full length of MoGLN1, MoGLN2, and MoGLN3 genes and their respective $2.3 \mathrm{~Kb}$ native promoters were amplified with primer pairs $\mathrm{G} \ln 1 \mathrm{com} F / R, G \ln 2 \mathrm{com} F / R$, and $\mathrm{Gln} 3 \mathrm{comF} / \mathrm{R}$ (Table S1). The resulting PCR products were cloned in pKNTG vector containing neomycin resistance. Each construct was transformed in its respective mutant protoplast.

\subsection{Appressorium Formation, Penetration, and Infection Assays}

Conidia collected from 10-day-old rice-bran culture were adjusted to $\left(5 \times 10^{4} \mathrm{spores} / \mathrm{mL}\right)$ using sterilized double-distilled water with $0.02 \%(v / v)$ Tween-20 solution. Appressorium formation assays were performed by adding $20 \mu \mathrm{L}$ of conidial suspension from Guy11; $\Delta M \circ g \ln 1$ and $\Delta M o g \ln 3$ strains were on an artificial hydrophobic coverslip and incubated in darkness at $28^{\circ} \mathrm{C}$. Appressorium formation was then examined at $4 \mathrm{~h}, 8 \mathrm{~h}, 12 \mathrm{~h}$, and $24 \mathrm{~h}$ time intervals. Conidia germination and appressorium formation on inductive surfaces were measured as described previously [45].

To observe the formation of the appressorium-like structure, mycelial plugs from Guy11, $\Delta M o g \ln 1, \Delta M o g \ln 2$, and $\Delta M o g \ln 3$ strains were inoculated on artificial cover slips and 10-day-old barley leaves; appressorium-like structure formation was then observed after $30 \mathrm{~h}$.

Rice infection was performed by spraying 3-week-old rice (Oryzae sativa cv. CO39) seedlings with the Guy11, $\Delta M o g \ln 1$, and $\Delta M o g \ln 3$ strain conidial suspension $(5 \times$ $10^{4}$ spores $/ \mathrm{mL}$ ). The infected plants were incubated in a humid chamber at $28{ }^{\circ} \mathrm{C}$ for $24 \mathrm{~h}$ in darkness and later transferred to a $12 \mathrm{~h}$ photoperiod chamber. Leaves were then imaged 7 days after infection.

For the barley infection assay, mycelial plugs derived from Guy11, $\Delta M \circ g \ln 1, \Delta M o g \ln 2$, and $\Delta M \operatorname{Mog} \ln 3$ were incubated on 10 -day-old barley leaves at $28^{\circ} \mathrm{C}$ for $24 \mathrm{~h}$ in darkness. Later, they were transferred to light conditions and imaged after 7 days.

To observe penetration and invasive hyphal growth, $10 \mu \mathrm{L}$ of Guy11, $\Delta M \operatorname{Mog} \ln 1$, and $\Delta M o g \ln 3$ conidial suspension $\left(5 \times 10^{4}\right.$ spores $\left./ \mathrm{mL}\right)$ was repeatedly dropped on 10-day-old barley leaves. The infected leaves were then incubated for $30 \mathrm{~h}, 48 \mathrm{~h}$, and $72 \mathrm{~h}$ at $28{ }^{\circ} \mathrm{C}$ under humid conditions; penetration and invasive hyphal growth were examined using a microscope.

\subsection{Nucleic Acid Manipulation, Southern Blotting Analysis, and qRT-PCR}

DNA extraction was performed using cetyltrimethylammonium bromide (CTAB) [46], gel electrophoresis, enzyme digestion, and ligation, and Southern blot hybridization was performed using standard procedures [47]. Probe labelling, hybridization, and detection were performed with a DIG High Prime DNA Labeling and Detection Starter Kit (Roche Applied Science, Penzberg, Germany).

Total RNA was isolated from frozen fungal mycelia and rice leaves using a Magen universal RNA kit as previously described [48]. To measure the relative abundance of gene transcripts, RNA was extracted from mycelia cultured in CM liquid medium for 3 days at $28{ }^{\circ} \mathrm{C}$ in an orbital shaker $(110 \mathrm{rpm})$. To measure the relative abundance of MoGLN, MoGLN2, and MoGLN3 transcripts during fungal developmental stages, the total RNA samples were extracted from mycelia grown in $\mathrm{CM}$ liquid medium, conidia, and rice leaves inoculated with the conidia of Guy11 $\left(1 \times 10^{8}\right.$ spores $\left./ \mathrm{mL}\right)$ for 8, 24, 48 and $72 \mathrm{~h}$.

Total RNA for all the samples was extracted using a Magen universal RNA kit, as described previously [48]. For RT-PCR and quantitative real time RT-PCR (qRT-PCR), $5 \mathrm{mg}$ of total RNA was converted to cDNA using HiScript ${ }^{\circledR} 11 \mathrm{Q}$ select RT supermix (vazyme). The qRT-PCR data were generated with an Eppendorf Realplex2 master cycler (Eppendorf AG 223341, Hamburg, Germany). Thermocycler conditions were as follows: 2 min at $95^{\circ} \mathrm{C}$, followed by 40 cycles of $95^{\circ} \mathrm{C}$ for $15 \mathrm{~s}, 60^{\circ} \mathrm{C}$ for $30 \mathrm{~s}$, and lastly, the melting curve stage of $95{ }^{\circ} \mathrm{C}$ for $15 \mathrm{~s}, 60{ }^{\circ} \mathrm{C}$ for $15 \mathrm{~s}$, and $95{ }^{\circ} \mathrm{C}$ for $15 \mathrm{~s}$. The stable expression actin 
gene (MGG_03982) was used as internal control, and three independent replicates were performed for each experiment; the qRT-PCR primers used are listed in Table S1. Data analysis was performed using the delta delta-CT $(2-\Delta \Delta \mathrm{CT})$ method, as described in [49].

\subsection{Western Blot Assays}

The wild-type Guy11 and the mutant strains were grown in liquid CM medium at $28^{\circ} \mathrm{C}$ for 4 days with agitation at $110 \mathrm{rpm}$. Total protein was extracted from mycelia as described previously [50]. The intensity of the signal corresponding to phosphorylated Mps1 was detected by binding of phospho-p44/42 MAPK (ERK1/2) (Thr202/Tyr204) (D13.14.4E) Rabbit mAb and p44/42 MAPK (ERK1/2) antibodies (Cell Signaling Technology, Beverly, MA, USA).

\subsection{RNA Isolation, Library Construction, and Sequencing}

Total RNA from Guy11 and $\Delta M o g \ln 2$ mutants was extracted using a Magen Kit as described previously [48]. The RNA integrity was assessed using the RNA Nano 6000 Assay Kit of the Bioanalyzer 2100 system (Agilent Technologies, California, CA, USA). cDNA libraries were constructed, and Illumina sequencing was performed on (Novaseq platform). Isolation of poly(A) mRNA from total RNA and construction of cDNA libraries were performed according to methods described previously [51]. After removing low-quality raw reads, the clean reads from each library were aligned to the transcript sequences of the Magnaporthe oryzae isolate 70-15 using bowtie2 (v2.3.4.1) [52], and the average mapping rate was $80.41 \%$. The expression abundance was calculated by RSEM (v1.3.1) [53] with default parameters, and the TPM values of transcripts were exported to DESeq2 (v1.29.16) [54] for differential expression analysis. A gene was defined as being a differentially expressed gene (DEG) in the case of:

i. a minimum 2-fold difference in gene expression between the control Guy11 and the $\Delta M o g \ln 2\left(\mid \log _{2} \mathrm{FCl}>1\right)$;

ii. a maximum false discovery rate (FDR) of 0.01 (FDR $<0.01)$.

\subsection{High-Performance Liquid Chromatography (HPLC) Assays}

Samples for glutamine for other amino acid tests were prepared by culturing the Guy11 and the $\Delta M o g \ln 1, \Delta M o g \ln 2$, and $\Delta M o g \ln 3$ at $28^{\circ} \mathrm{C}$ in liquid $\mathrm{CM}$ medium for three days and transferring to minimal medium for an additional two days with agitation (110 rpm). The strains were then filtered out, rinsed with sterilized double-distilled water, and frozen in liquid nitrogen. The dried hyphae tissues from the respective strains were ground into powders using a mortar and pestle. The grinded hyphae generated from the Guy11 and the MoGLN mutants were separately weighed into $2 \mathrm{~mL}$ Eppendorf tubes (EP-tubes) containing $50 \mu \mathrm{mol} / \mathrm{L}$ BTI acetonitrile. A total of $25 \mu \mathrm{L}$ of pyridine aqueous solution $(50 \mu \mathrm{mol} / \mathrm{L})$ was added and mixed well. The samples were then incubated at $50{ }^{\circ} \mathrm{C}$ for $4 \mathrm{~h}$. A total of $200 \mu \mathrm{L}$ of $6 \mathrm{~mol} / \mathrm{HCl}$ was then added, followed by hydrolyzation at $110^{\circ} \mathrm{C}$ for $24 \mathrm{~h}$. After the hydrolysis was completed, samples were dried with nitrogen and hydrolyzed with $100 \mu \mathrm{L}$ of acetonitrile-pyridine-triethylamine-water (10:5:2:3) buffer. Then, $20 \mu \mathrm{L}$ PITC (phenyl isothiocyanate) was added and incubated at $50^{\circ} \mathrm{C}$ for $1 \mathrm{~h}$. Following this, $250 \mu \mathrm{L}$ of the samples were pipetted into a clean $2.0 \mathrm{~mL}$ EP tube, containing $750 \mu \mathrm{L}$ $0.02 \mathrm{~mol} / \mathrm{L} \mathrm{HCL}$ and $200 \mu \mathrm{L}$ N-hexane to remove impurities. The lower layer was then carefully transferred to the new $2.0 \mathrm{~mL}$ EP tubes for detection of glutamine. The quantifying services were performed and completed by Qingdao Sci-tech Innovation Quality Testing Co. Ltd., Qingdao, China.

For detection of other amino acids in Guy11 and $\triangle M o g \ln 2$ mutants, ACQUITY UPLC ${ }^{\circledR}$ BEH C18 column $(2.1 \times 100 \mathrm{~mm}, 1.7 \mu \mathrm{m}$, Waters, Milford, MA, USA) model HPLC instrument was used. Injection volume was $5 \mu \mathrm{L}$, and column temperature was $40{ }^{\circ} \mathrm{C}$. Mobile phase A used $10 \%$ methanol water (containing $0.1 \%$ formic acid) and mobile phase B used $50 \%$ methanol water (containing $0.1 \%$ formic acid). The gradient elution conditions were $0 \sim 6.5 \mathrm{~min}, 10 \sim 30 \%$ mobile phase B; $6.5 \sim 7 \mathrm{~min}, 30 \sim 100 \%$ mobile phase $\mathrm{B} ; 7 \sim 8 \mathrm{~min}, 100 \%$ mo- 
bile phase $\mathrm{B}$; 8 8.5 $\mathrm{min}, 100 \sim 10 \%$ mobile phase B; 8.5 12.5 $\mathrm{min}, 10 \%$ mobile phase B. Flow rate was $0 \sim 8.5 \mathrm{~min}, 0.3 \mathrm{~mL} / \mathrm{min}$ and $8.5 \sim 12.5 \mathrm{~min}, 0.3 \sim 0.4 \mathrm{~mL} / \mathrm{min}$. Mass spectrometry conditions were as follows: electrospray ionization (ESI) source, positive ionization mode. The ion source temperature was $500{ }^{\circ} \mathrm{C}$, the ion source voltage was $5500 \mathrm{~V}$, the collision gas was 6 psi, the curtain gas was 30 psi, and the atomization gas and auxiliary gas were both 50 psi. The quantifying services were performed and completed by Fuzhou Beiruisi Biotechnology Co. Ltd., Fuzhou, China.

\subsection{Microscopy}

To observe conidiophore development, conidia shapes, appressorium formation on inductive surfaces, appressorium penetration, and invasive hyphae development, an Olympus DP80 light microscope (Tokyo, Japan) was used, while GFP localization assays were examined using a confocal microscope equipped with Nikon A1 plus instrument (Nikon, Tokyo, Japan).

\subsection{Bioinformatic Analysis}

To identify MoGln1, MoGln2, and MoGln3 in the M. oryzae, the S. cerevisiae Gln1 amino acid sequence was used to perform a blastP search in the M. oryzae genome KEEG database (http: / / www.kegg.jp/kegg-bin/show_organism?org=mgr, accessed on 10 January 2020). The Gln1, Gln2, and Gln3 amino acid ortholog sequences from different fungi were obtained from (www.ncbi.nlm.nih.gov/blast, accessed on 10 January 2020) using the blast algorithm [55]. Domains were predicted by Pfam (http:/ / pfam.janelia.org/, accessed on 10 January 2020) and presented using IBIS 1.0.3 software [56]. Sequence alignment was performed using MEGA v6, while phylogenetic tree was generated using the MaximumLikelihood method, with branches of the tree tested with 1000 bootstrap replicates. The accession number for amino acid sequences used for phylogenetic analysis is as follows: MoGLN1 (XP_003709618); MoGLN2 (XP_003719336); MoGLN3 (XP_003721264); NcGLN1 (XP_960904); SsGLN1 (XP_001588876); FoGLN1 (XP_018240222); FgGLN1 (XP_011319217); TrGLN1 (XP_006967001); AfGLN1 (XP_023088587); AniGLN1 (XP_661763); UmGLN1 (XP_011390105); ScGLN1 (ONH79708); FfGLN1 (XP_023431521); AfGLN3 (RAQ56449); FgGLN3 (XP_011315791); TrGLN3 (XP_006967843); UmGLN3 (XP_011392295); AniGLN3 (XP_664258); NcGLN3 (XP_965073); SsGLN1 (XP_001593468).

\section{Results}

\subsection{Identification of Glutamine Synthetase in M. oryzae}

To obtain sequences for $M$. oryzae glutamine synthetase genes, referred to here as MoGLN, the amino acid sequence of glutamine synthetase gene (GLN1) from Saccharamycess cerevisiae was used to conduct a blastP search in Kyoto Encyclopedia of Genes and Genome (KEGG) resource section for M. oryzae (http: / / www.kegg.jp/kegg-bin/show_organism? org=mgr, accessed on 10 January 2020). Three putative amino acid sequences that encode glutamine synthetase were identified and were named, based on a previous study, as MoGln1 (MGG_06888), MoGln2 (MGG_14279), and MoGln3 (MGG_02538) [57]. The three obtained MoGln amino acids were used for a blastP search to identify glutamine synthetase amino acid sequences in other fungi in the Fungi and Oomycetes genomics resources database (http:/ / fungidb.org/fungidb / , accessed on 10 January 2020) and National Centre of Biotechnology Information (https:/ / www.ncbi.nlm.nih.gov/, accessed on 10 January 2020). The retrieved amino acid sequences were then used to conduct Pfambased domain prediction. Results obtained showed that Gln1 and Gln2 contained two conserved domains - glutamine synthetase, a catalytic domain, and glutamine synthentase, a beta-Grasp domain — while Gln3 contained a single glutamine synthentase-beta-Grasp domain-and this domain was conserved in fungi (Figure 1A-C). Phylogenetic analysis revealed that MoG1n1, MoG1n2, and MoG1n3 shared a close ancestor with Gln1, G1n2, and G1n3 of Neurospora crassa (Nc), respectively (Figure 1A-C). 

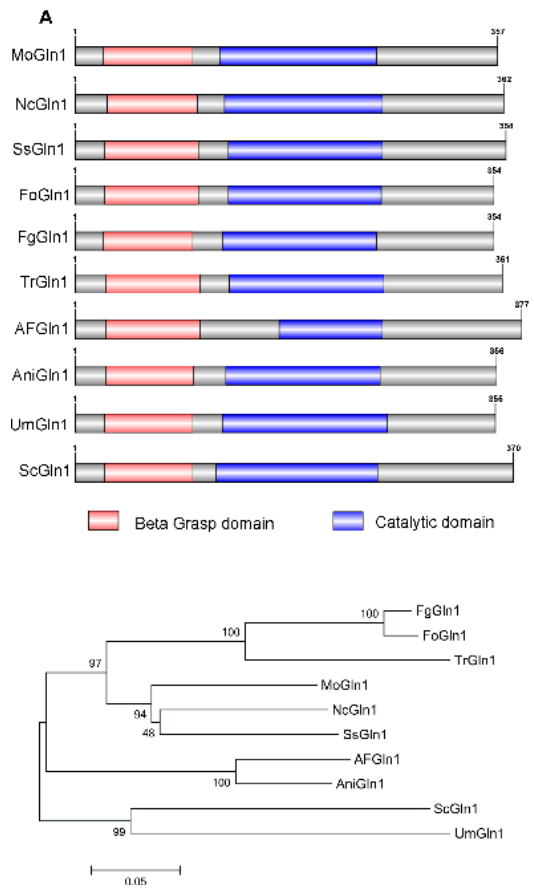

B
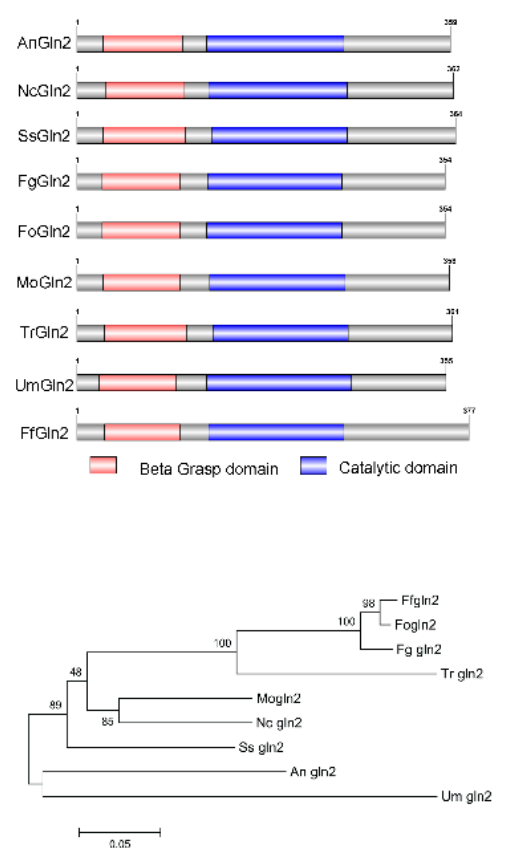

C
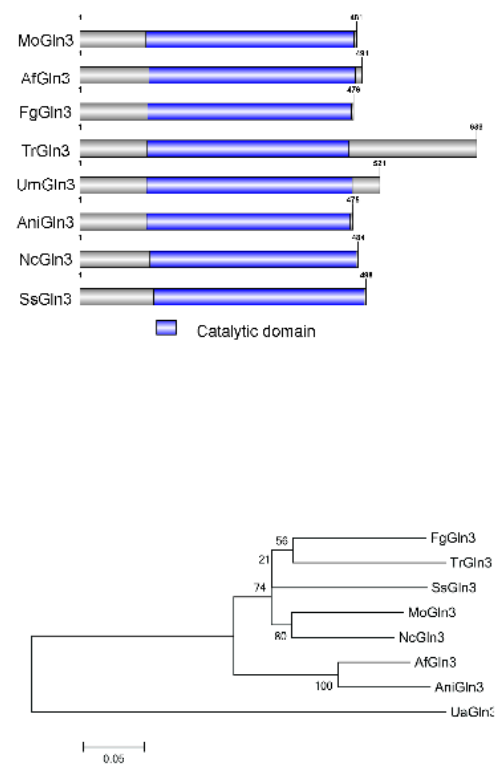

Figure 1. Domain architecture and phylogeny of $M$. oryzae glutamine synthetase in different fungi: (A) Gln1 domain architecture and maximum likelihood based phylogenetic outlook in different fungi; (B) Gln2 domain architecture and maximum likelihood phylogenetic analysis in fungi groups; (C) Domain architecture of Gln3 and the maximum likelihood phylogeny in different fungi. Maximum likelihood phylogeny for the glutamine synthetase amino acids of different fungi was tested with 1000 bootstrap replicates.

\subsection{Expression of MoGLN Genes at Different Developmental Stages of M. oryzae}

It was initially assumed that through-checking the expression pattern of the three MoGLN genes at various developmental stages of the fungus would provide information on their likely roles. Using the WT strain, we quantified the expression level of these genes at conidia and in the planta stage $(8 \mathrm{~h}, 24 \mathrm{~h}, 48 \mathrm{~h}$, and $72 \mathrm{~h})$. The Guy11 mycelia stage was used as a control, and in the planta stages, 21-day-old rice leaves were sprayed with Guy11 spores. The expression of MoGLN1 was found to be higher at the late infection stages of fungus, with the fold increases of $-0.7,0.1,-0.5,5.5$, and 4.0 at sporulation, $8 \mathrm{~h}, 24 \mathrm{~h}, 48 \mathrm{~h}$, and $72 \mathrm{~h}$, respectively (Figure 2A). For MoGLN2, we noted an elevated expression at the sporulation stage, with fold increases of 2.0, 1.0, 0.4, -0.6 , and -0.8 at conidiation, $8 \mathrm{~h}, 24 \mathrm{~h}, 48 \mathrm{~h}$, and $72 \mathrm{~h}$, respectively (Figure 2B). Finally, our stage-specific qPCR analysis established that the transcripts levels of MoGLN3 were high at the early infection stage, with fold increases of $2.4,3.8,0.8,0.6$, and 1.0 at the asexual stage, $8 \mathrm{~h}, 24 \mathrm{~h}$, $48 \mathrm{~h}$, and $72 \mathrm{~h}$, respectively (Figure 2C). To validate the exact functions of the three MoGLN genes, their respective deletion mutants were generated, and phenotype was characterized.

\subsection{Generation of $\Delta M o g \ln 1, \Delta M o g \ln 2$, and $\Delta M o g \ln 3$ Deletion Strains}

To establish the exact roles of the MoGLN genes in development and pathogenicity of the rice blast fungus, we generated their respective deletion mutants using a homologous recombination approach by replacing each of MoGLN1, MoGLN2, and MoGLN3 open reading frame $(\mathrm{ORF})$ with the hygromycin phosphotransferase $(\mathrm{HPH})$ gene. To generate the gene deletion constructs, the upstream (A fragment) and downstream (B fragment) flanking regions of MoGLN1, MoGLN2, and MoGLN3 were amplified and ligated with $5^{\prime}$ and $3^{\prime}$ split parts of a hygromycin-resistant gene. Each of the constructs was separately transformed in Guy11 protoplast and screened on TB3 medium containing hygromycin resistance. Putative transformants for MoGLN1, MoGLN2, and MoGLN3 deletions were screened by PCR with gene-specific ORF primer pairs (Table S1), and successful deletion of 
MoGLN1, MoGLN2 and MoGLN3 was subsequently confirmed using southern blot assays. Results obtained after confirmation assays showed MoGLN1, MoGLN2, and MoGLN3 open reading frame (ORF) were successfully replaced with a single integration of hygromycin phosphotransferase $(H P H)$ to generate $\Delta M o g \ln 1$ (Figure $3 \mathrm{~A}, \mathrm{~B}), \Delta M o g \ln 2$ (Figure $3 \mathrm{C}, \mathrm{D}$ ), and $\Delta M o g \ln 3$ (Figure $3 \mathrm{E}, \mathrm{F}$ ).

A

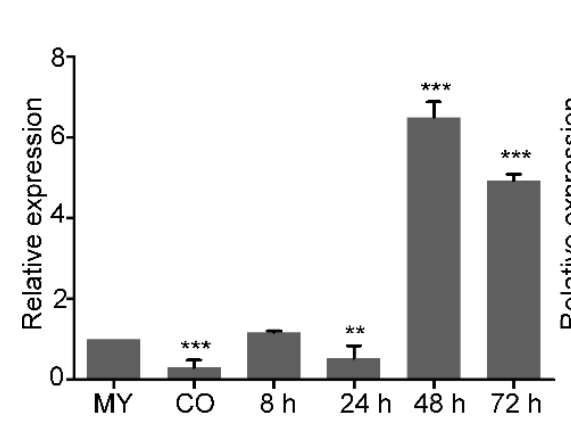

B

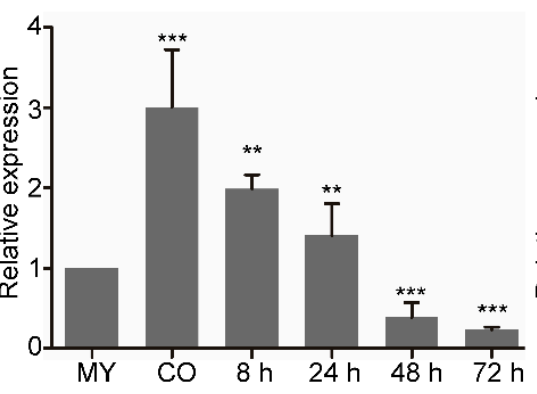

\section{C}

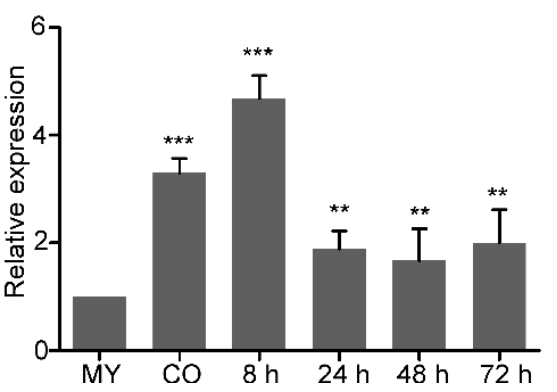

Figure 2. Phase-specific expression of the three MoGLN genes at various development stages of M. oryzae. (A) phasespecific expression of MoGLN1; (B) phase-specific expression of MoGLN2; (C) phase-specific expression of MoGLN3. The phase-specific expression of the three MoGLN genes was quantified by quantitative real-time (QRT)-PCR after synthesis of cDNA in each developmental stage. The ACTIN gene (MGG_03982) was used for internal control for normalization, and the expression level of each gene at the mycelial stage was considered 1 for further comparisons. The qPCR results were obtained from three independent biological replications with three technical replicates. Error bars represent standard deviations. Asterisks indicate statistically significant differences ${ }^{* *}, p<0.01$; ${ }^{* * *}, p<0.001$; one-way ANOVA was used to analyze data with Tukey's multiple-comparison test in GraphPad Prism 8).

A

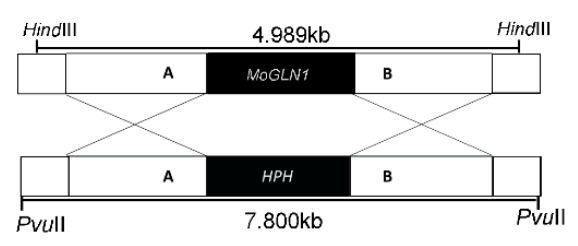

B
C

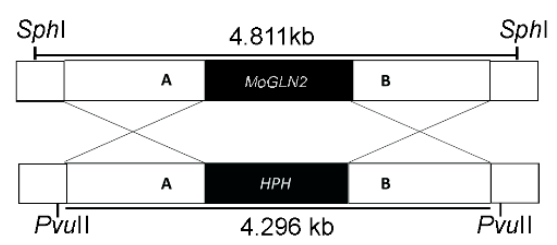

D

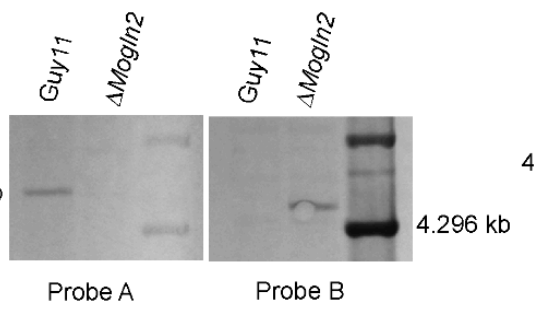

E

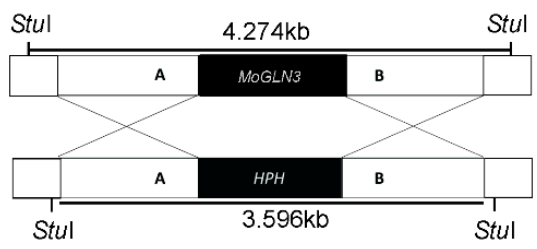

$\mathbf{F}$

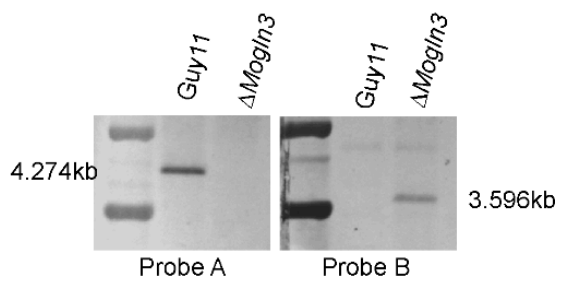

Probe A Probe $\mathrm{B}$

$7.800 \mathrm{~kb}$

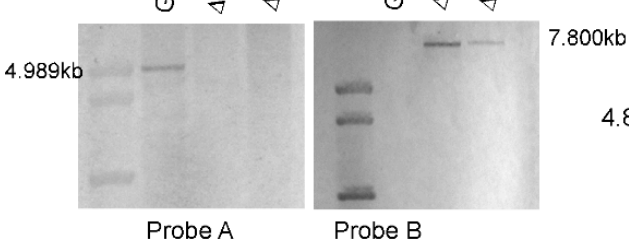

Figure 3. Southern blot analysis to confirm MoGLN deletion mutants. (A,B) Sketch representation of deletion of MoGLN1 in the M. oryzae genome and southern blot analysis of the gene knockout mutants and WT Guy11 via MoGLN1 ORF probe A and hygromycin phosphotransferase $(H P H)$ probe B. (C,D) Sketch representation of deletion of MoGLN2 in M. oryzae genome and southern blot analysis of the gene knockout mutant and WT Guy11 via MoGLN2 ORF probe A and hygromycin phosphotransferase $(H P H)$ probe B. (E,F) Sketch representation of deletion of MoGLN3 in the M. oryzae genome and southern blot analysis of the gene knockout mutant and the WT Guy11 using MoGLN3 ORF probe A and hygromycin phosphotransferase $(H P H)$ probe $\mathrm{B}$.

\subsection{MoGLN2 Contributes to Vegetative Growth in M. oryzae}

To investigate the contribution of MoGLN genes in vegetative growth of M. oryzae, we cultured the three respective MoGLN mutants on CM (complete medium), MM (minimal medium), OTM (oatmeal medium), and SDC (straw decoction and corn medium) 
and observed their growth. After eight days of inoculation, we established no significant difference in mycelial growth in terms of colony diameter between the wild-type Guy11, $\Delta M o g \ln 1$, and $\Delta M o g \ln 3$ on four different types of medium (Figure 4A,B). However, the growth of $\triangle M o g \ln 2$ mutants was remarkably reduced in CM, OTM, and SDC, and the mutant failed to grow on MM medium (Figure $4 \mathrm{~A}, \mathrm{~B}$ ). In addition, the $\Delta M o g \ln 2$ exhibited poor development of aerial hyphal on SDC and OTM when compared to the wild-type Guy11, $\Delta M o g \ln 1$, and $\Delta M o g \ln 3$ strains (Figure 4A,B). Introduction of MoGLN2 gene into the $\Delta M \operatorname{og} \ln 2$ mutant restored the growth defects of $\Delta M \operatorname{Mog} \ln 2$ on CM, MM, OTM, and SDC. These results indicated that MoGLN2 is required for proper vegetative growth in M. oryzae.

A

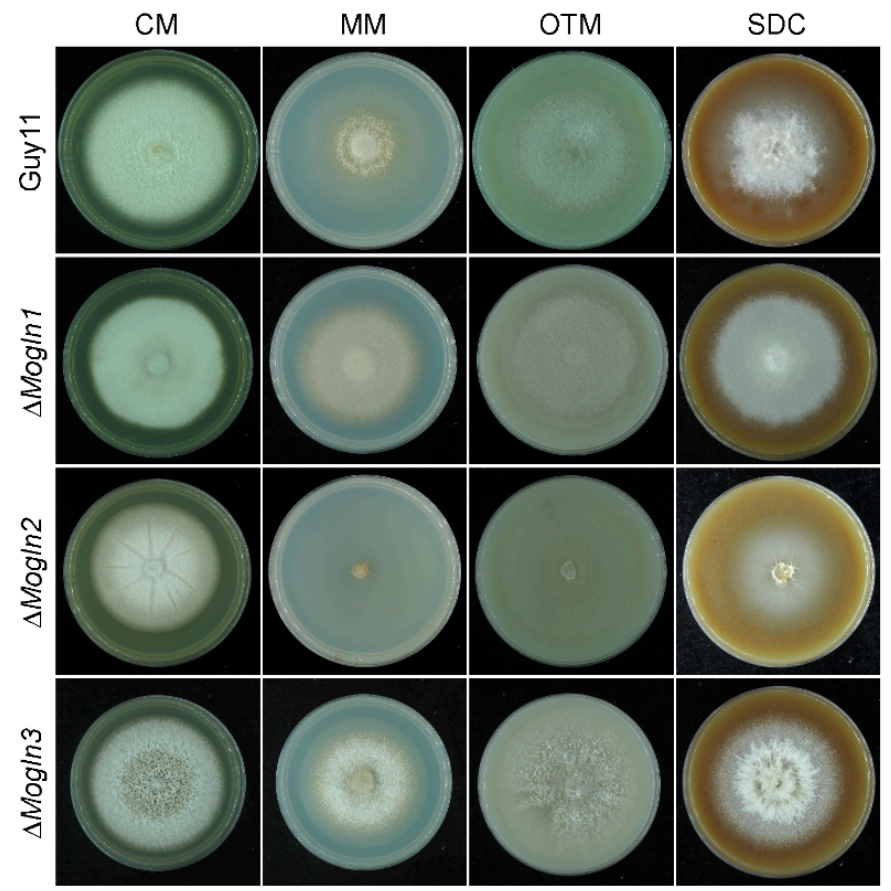

B

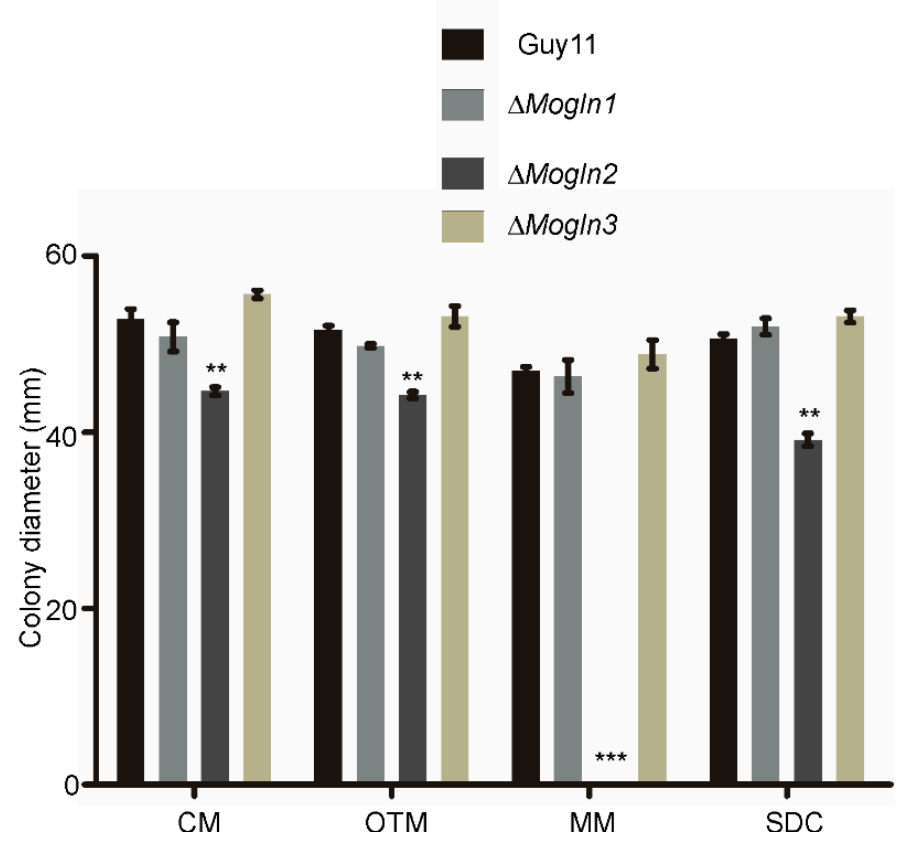

Figure 4. MoGLN2 is required for vegetative growth in M. oryzae. (A) Photographs showing radial and aerial hyphal growth of the wild-type (WT) and the three mutants. Mycelial plugs inoculated on CM, MM, OTM, and SDC were cultured in the dark at $28^{\circ} \mathrm{C}$, and photograph taken after eight days. (B) Bar graphs showing the difference in radial growth between the WT and the three MoGLN mutants. The error bar represents the standard deviation of three independent replicates, while the double asterisk shows significant difference ${ }^{* *}, p<0.01$; ${ }^{* *}, p<0.001$; one-way ANOVA was used to analyze data with Tukey's multiple-comparison test in GraphPad Prism 8).

\subsection{Glutamine Auxotroph in Rice Blast Fungus Occurs via Inactivation of MoGLN2}

In Aspergillus nidulans, deletion of the glutamine synthetase gene resulted in the mutant cells requiring glutamine for growth in MM medium [34]. Because the $\Delta M o g \ln 2$ mutant was attenuated in MM, we speculated the mutant cells lacked sufficient glutamine levels required for growth. To test this idea, we first tested the growth of $\Delta M o g \ln 2$ in MM medium supplemented with different concentrations of glutamine $(0.1 \mathrm{mM}, 0.625 \mathrm{mM}, 1.25 \mathrm{mM}$, $2.5 \mathrm{mM}$, and $5 \mathrm{mM}$ ). Our results showed that exogenous glutamine could restore growth of $\Delta M o g \ln 2$ on MM medium, with more aerial hyphal being observed at high concentrations of glutamine (Figure 5A). Since the growth defect of $\Delta M o g \ln 2$ on MM medium was attributed to insufficient glutamine levels, we detected intracellular glutamine in the mycelia of the three MoGLN mutants. No significant change in glutamine levels was recorded in $\Delta M o g \ln 1$, while glutamine levels were significantly lower and higher in $\Delta M o g \ln 2$ and $\Delta M o g \ln 3$ strains, respectively (Figure $5 \mathrm{~B}$ ). We then sorted to determine if deletion of one MoGLN gene affected the expression of the remaining two genes. We observed an up- 
regulation of MoGLN2 in $\triangle M o g \ln 1$ mutant, with expression of MoGLN3 being unaffected (Figure 5C). The expression level of MoGLN1 was higher in the $\Delta M o g \ln 2$ mutant, with no detectable change in expression of MoGLN3 (Figure 5D). Lastly, both MoGLN1 and MoGLN2 were up-regulated in $\triangle M o g l n 3$ mutant (Figure 5E). These expression patterns showed that glutamine levels in $\triangle M o g \ln 1$ were from $M o G L N 2$, low levels of glutamine in the $\Delta M o g \ln 2$ mutant were from MoGLN1, and the highest glutamine levels in $\Delta M o g \ln 3$ were from MoGLN1 and MoGLN2. These results suggest that both MoGLN1 and MoGLN2 could be involved in de novo glutamine biosynthesis. However, the level of glutamine produced by MoGLN1 is not sufficient enough to sustain normal cellular function. Further evidence for the involvement of MoGLN1 in glutamine biosynthesis was reported in $\triangle M o a s d 4$ after glutamine levels were significantly lowered in $\triangle$ Moasd4 upon deletion of MoGLN1 in $\Delta$ Moasd4 [57]. Overall, we conclude that glutamine auxotroph in rice blast fungus only occurs via deletion of MoGLN2.

A
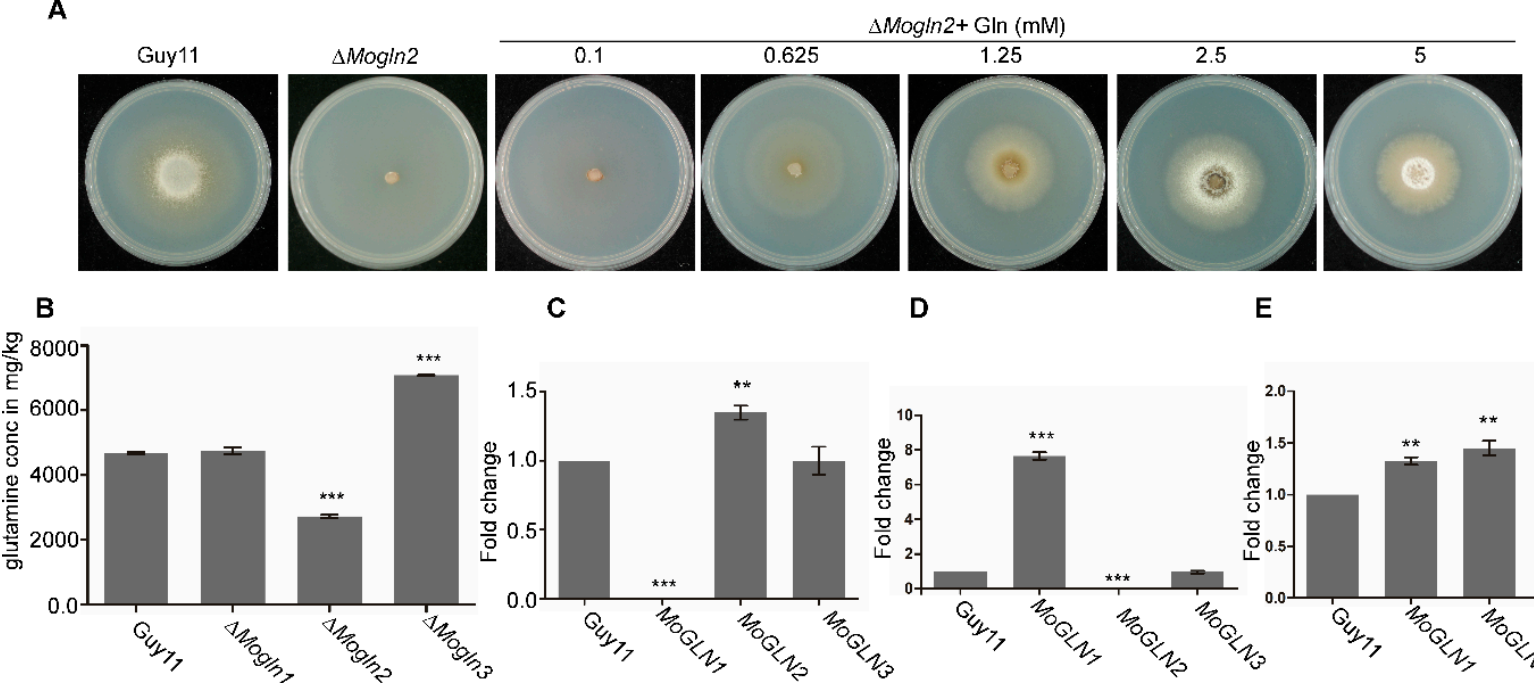

D

E

Figure 5. Exogenous glutamine restores growth defects of $\Delta M o g \ln 2$ on MM medium. (A) Radial growth of $\Delta M o g \ln 2$ mutant on MM medium supplemented with different concentrations of glutamine. The experiment was repeated three times with similar results obtained. (B) Statistical representation of intracellular glutamine levels detected in WT and the three MoGLN mutants. Error bars represent standard deviations obtained from two independent tests. Data were analyzed using GraphPad Prism 8; asterisks indicate statistically significant differences ${ }^{* *}, p<0.01$; ${ }^{* *}, p<0.001$; based on one-way ANOVA with Tukey's multiple-comparison test). (C) Graph showing the expression of MoGLN2 and MoGLN3 in $\triangle M o g \ln 1$ mutant; (D) graphical representation of expression pattern of MoGLN1 and MoGLN3 in the $\triangle M o g \ln 2$ mutant; (E) expression pattern of MoGLN1 and MoGLN2 in $\triangle M o g l n 3$ mutant. The actin gene was used as a control. Data for statistical analysis were obtained after performing three independent biological replicates. Error bars represent standard deviations. Asterisks indicate statistically significant differences $\left({ }^{* *}, p<0.01 ;{ }^{* * *}, p<0.001\right.$; one-way ANOVA was used to analyze data with Tukey's multiple-comparison test in GraphPadPrism 8).

\subsection{MoGLN2 Is Required for Asexual Reproduction in M. oryzae}

To determine the roles of the three MoGLN2 genes in sexual reproduction in rice blast fungus, the wild-type Guy11 and the three mutant strains were cultured on sporulation rice bran medium for 10 days, and then conidiophore development and conidia formation evaluated. The wild-type strain, $\Delta M o g \ln 1$, and $\Delta M o g \ln 3$ produced similar conidiophores and an equal number of spores (Figure 6A,B). No conidia or conidiophores was formed in the cultures of $\Delta M o g \ln 2$ mutants (Figure $6 \mathrm{~A}, \mathrm{~B}$ ). Since rice bran medium could not initiate conidiation in $\Delta M o g \ln 2$, we tried different conidiation media, including OTM and SDC. Neither of these activated conidiation in the $\Delta M o g \ln 2$ mutant. As glutamine synthetase catalyzes the biosynthesis of glutamine, we supplemented rice bran, OTM, and SDC media with different concentrations of glutamine $(1 \mathrm{mM}, 2 \mathrm{mM}, 5 \mathrm{mM}, 10 \mathrm{mM}, 20 \mathrm{mM}$, $40 \mathrm{mM}$, and $60 \mathrm{mM}$ ). None of these glutamine concentrations rescued the conidiation 
defects in the $\Delta M \operatorname{Mog} \ln 2$ mutant. We then performed quantitative real-time PCR (qRT-PCR) analysis to check the transcript levels of conidiation-related genes, including COS1, COM1, CON6, CON7, HOX6, HOX7, and STUA. The expression of these genes was found to be significantly reduced in the $\triangle M o g \ln 2$ mutant (Figure $6 \mathrm{C}$ ), indicating that MoGLN2 regulates conidiogenesis in rice blast fungus through controlling the expression of conidiation-related genes. In summary, these results shows that MoGLN2 plays an important role in asexual reproduction in rice blast fungus.

A
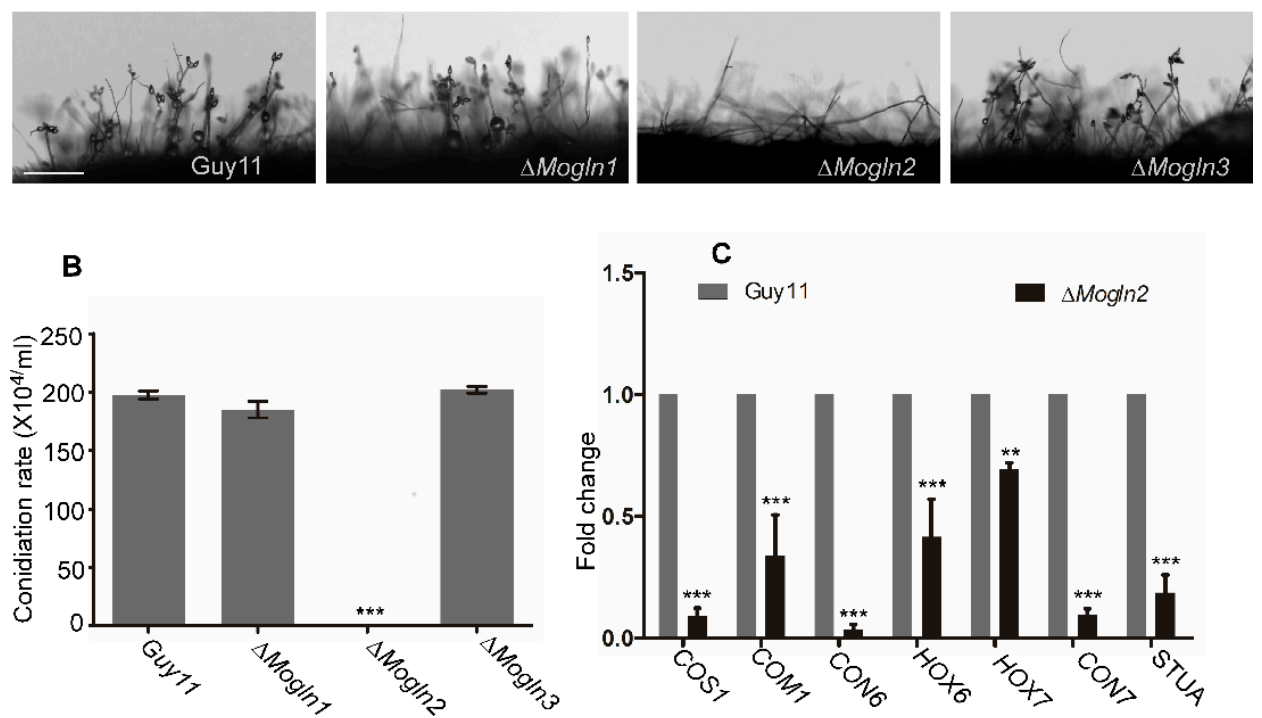

Figure 6. MoGln2 plays an important role in asexual reproduction in M. oryzae. (A) Represents conidiophore development and spore formation capacity of strains cultured on rice bran medium for 10 days. Bar, $10 \mu \mathrm{m}$. (B) Graph showing quantification of spores from Guy11, $\Delta M o g \ln 1, \Delta M o g \ln 2, \Delta M o g \ln 3$ strains on rice bran medium. The $\Delta M o g \ln 2$ mutant failed to produce spores. (C) Quantitative RT-PCR analysis showing the expression of conidiation-related genes in the WT and $\Delta M o g \ln 2$ mutants. The expression was normalized actin gene (MGG_03982). Results are means obtained from three independent replicates. Error bars represents standard deviations. Asterisks indicate statistically significant differences (**, $p<0.01 ;{ }^{* * *}, p<0.001$; one-way ANOVA was applied to analyze data with Tukey's multiple-comparison test in GraphPad Prism 8).

\subsection{MoGLN2 Is Important for Appressorium Formation in M. oryzae}

In rice blast fungus, infection occurs when $M$. oryzae spores land on the rice leaf surface and germinate into a specialized structure called appressorium. Besides rice leaves, appressorium also forms when $M$. oryzae spores encounter a hydrophobic surface, as it mimics the rice leaf surface. Because $\Delta M o g \ln 1$ and $\Delta M o g \ln 3$ produced spores, their respective spores alongside with those from the Guy11 wild strain were inoculated on an artificial hydrophobic surface, and spore germination and appressorium formation were examined at $4 \mathrm{~h}, 8 \mathrm{~h}, 12 \mathrm{~h}$, and $24 \mathrm{~h}$ time intervals. Both $\Delta M o g \ln 1$ and $\Delta M o g \ln 3$ produced normal appressoria that was indistinguishable from the wild-type strain (Figure 7A), thus confirming that MoGLN1 and MoGLN3 are not required for appressorium formation in M. oryzae. It has been reported that rice blast fungus can form appressoria from its hyphae [58]. We then evaluated appressorium formation using mycelia plugs of Guy11, $\Delta \operatorname{Mog} \ln 1, \Delta \operatorname{Mog} \ln 2$, and $\Delta M o g \ln 3$ strains by first observing appressorium-like structure formation on an artificial hydrophobic surface. After $24 \mathrm{~h}$, no appressorium-like structure formed on a hydrophobic surface inoculated with mycelia from $\Delta M o g \ln 2$; in contrast, the $\Delta M o g \ln 1$ and $\Delta M o g \ln 3$ formed appressorium-like structures similar to the Guy11 strain (Figure 7B). Furthermore, we examined appressorium-like structures on barley leaves inoculated with the three MoGLN mutants; similarly, $\triangle M o g \ln 2$ failed to form appressorium-like structures on barley leaves, but $\Delta M o g \ln 1$ and $\Delta M o g \ln 3$ and the Guy11 strain formed appressorium-like 
structures (Figure 7B). Collectively, we conclude that MoGLN2 is important for appressorium formation in rice blast fungus.

A
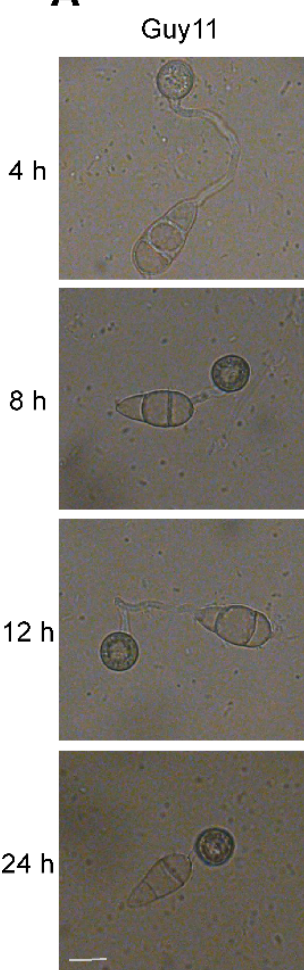

$\Delta M o g \ln 1$
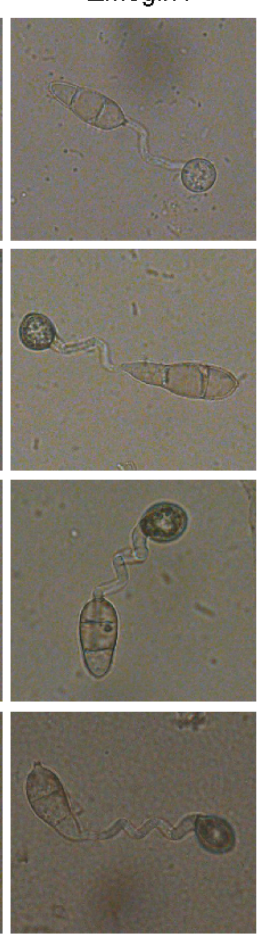

\section{$\Delta M o g \ln 3$}
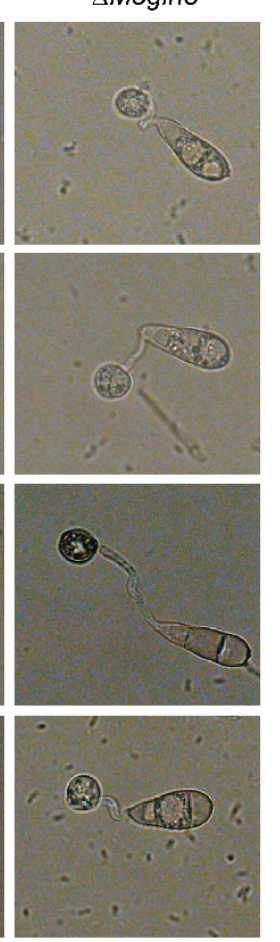

B
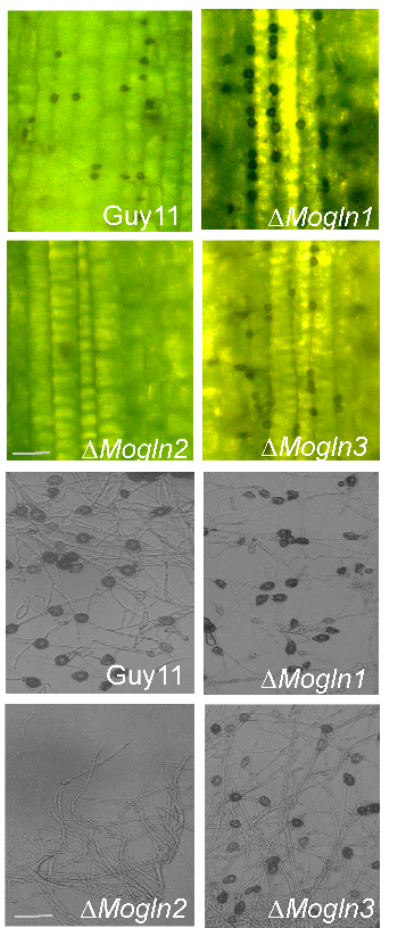

Figure 7. MoGln2 plays a crucial role in appressorium formation in rice blast fungus. (A) Bright field micrographs of the appressoria formed by WT, $\Delta M o g \ln 1$, and $\Delta M o g \ln 3$ mutants on inductive hydrophobic cover slips. Conidia from WT, $\Delta M o g \ln 1$, and $\Delta M o g \ln 3$ mutants were inoculated on a hydrophobic cover slip, and appressoria formation was observed at $4 \mathrm{~h}, 8 \mathrm{~h}, 12 \mathrm{~h}$, and $24 \mathrm{~h}$ time intervals. Scale bar $=10 \mu \mathrm{m}$. (B) An appressorium-like structure formed on a hydrophobic surface and barley leaves. Mycelia plugs derived from WT, $\Delta M o g \ln 1, \Delta M o g \ln 2$, and $\Delta M o g \ln 3$ were inoculated on 10-day-old barley leaves, and inductive hydrophobic cover slips; appressorium-like structure formation was observed after $30 \mathrm{~h}$. Scale bar $=10 \mu \mathrm{m}$. The $\Delta M o g h \mathrm{~h} 2$ mutant failed to form appressorium-like structures both on barley leaves and hydrophobic cover slips.

\subsection{MoGLN2 Is Essential for Full Virulence in M. oryzae}

To establish the role played by different subunits of glutamine synthase in the pathogenicity of rice blast fungus, we first examined the virulence of $\Delta M o g \ln 1, \Delta M o g \ln 2$, and $\Delta M o g \ln 3$ by inoculating their mycelial plugs on 10-day-old barley leaves. Seven days after inoculation, $\Delta \operatorname{Mog} \ln 2$ failed to cause disease symptoms both on intact and injured barley leaves, while the wild-type, $\Delta M \operatorname{og} \ln 1$, and $\Delta M o g \ln 3$ produced large similar blast lesions on barley leaves (Figure $8 \mathrm{~A}, \mathrm{~B}$ ). Since $\Delta M \log \ln 1$ and $\Delta M \operatorname{Mog} \ln 3$ strains could produce spores, we harvested the Guy11, $\Delta M o g \ln 1$, and $\Delta M o g \ln 3$ spores from 10-day-old rice bran cultures used to spray 3-week-old seedlings of the susceptible rice variety CO39. Our results showed that both $\Delta M o g \ln 1$ and $\Delta M o g \ln 3$ strains produced necrotic blast lesions on rice leaves similar to the wild-type strains (Figure 8C). Based on these results, we conclude that amongst the three MoGLN genes, MoGLN2 is solely involved in the pathogenicity of rice blast fungus. 

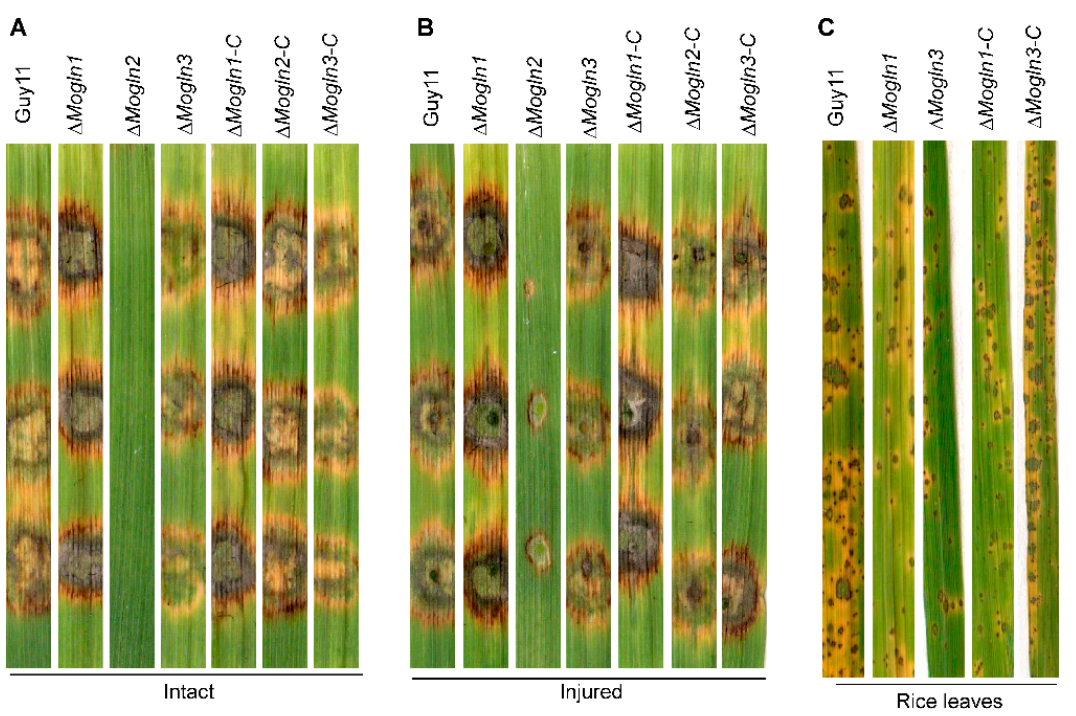

Figure 8. MoGLN2 plays an important role in promoting the infections of M. oryzae. (A,B) $\Delta M o g \ln 2$ failed to induce hyphae-mediated blast lesions on intact and injured barley leaves. (C) Rice leaves bearing blast lesions of $\Delta M o g \ln 1$ and $\Delta M o g \ln 3$ mutant spores. Both barley and rice leaf images were taken seven days post inoculation.

\subsection{MoGLN1 and MoGLN3 Are Not Involved in Appressorium Penetration and Infectious Hyphal Growth}

Appressorium penetration is an essential process that allows the fungus to get inside the host cell and cause infection. Appressorium-mediated penetration occurs when turgor pressure builds up within appressorium, which is used to breach the host surface [59]. Owing to the fact that $\Delta M o g \ln 1$ and $\Delta M o g \ln 3$ spores could form appressorium on the hydrophobic coverslip, we monitored appressorium penetration and subsequent invasive hyphal formation by inoculating conidia from Guy11, $\Delta M o g \ln 1$, and $\Delta M o g \ln 3$ on 10-dayold barley. After $30 \mathrm{~h}$ of inoculation, the majority of the appressoria of Guy11, $\Delta M \operatorname{Mog} \ln 1$, and $\Delta M o g \ln 3$ strains had penetrated the barley cells and started forming invasive hyphae (Figure 9). At $48 \mathrm{~h}$, the invasive hyphal of the three strains had spread and colonized the adjacent cells (Figure 9), clearly showing that deletion of either MoGLN1 or MoGLN3 did not affect these processes. These results indicate that both MoGLN1 and MoGLN3 are not required for appressorium penetration and invasive hyphal formation in rice blast fungus.
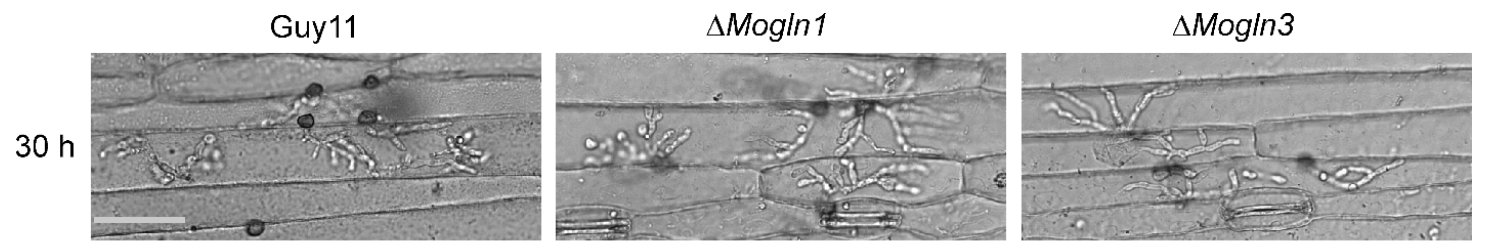

$48 \mathrm{~h}$
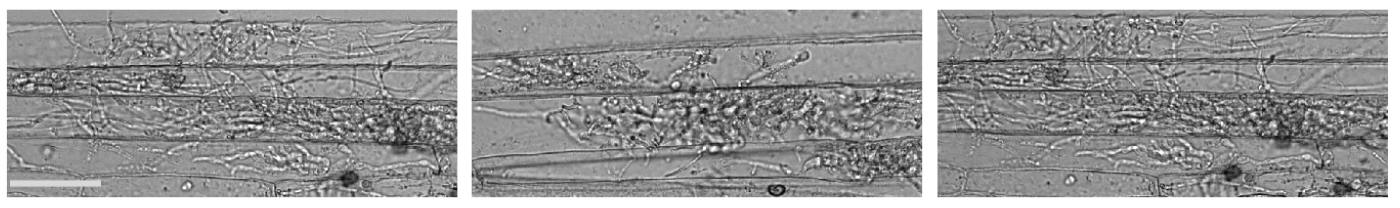

$60 \mathrm{~h}$
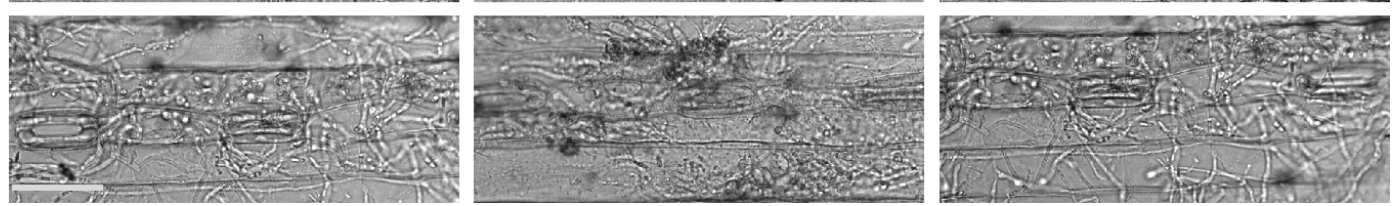

Figure 9. Bright field micrographs showing the development of invasive hyphal growth of WT, $\Delta M o g \ln 1$, and $\Delta M o g \ln 3$. Spore suspensions from Guy11, $\Delta M o g \ln 1$, and $\Delta M o g \ln 3$ strains were inoculated on 10-day-old barley leaves, and invasive hyphal growth was observed at $30 \mathrm{~h}, 48 \mathrm{~h}$, and $72 \mathrm{~h}$. Bar $=20 \mu \mathrm{m}$. 


\subsection{Cell Wall Integrity Is Impaired in the $\Delta$ Mogln2 Deletion Mutant}

To investigate the contribution of three MoGLN genes in fostering cell wall integrity in rice blast fungus, we first monitored and measured the vegetative growth of three MoGLN mutants on CM medium amended with cell wall stressors Calcofluor white (CFW) [60,61], Sodium Dodecyl Sulfate (SDS) [62,63], and Congo Red (CR) [64]. After eight days post inoculation, our results showed only $\Delta M \operatorname{Mog} \ln 1$ mutant was highly inhibited on plates containing CR. On SDS medium, both $\Delta M o g \ln 1$ and $\Delta M o g \ln 2$ were highly inhibited, while on CM medium containing CFW, $\Delta M o g \ln 2$ was slightly inhibited, with $\Delta M o g \ln 3$ being highly sensitive (Figure 10A,B). We performed additional tests to conclusively determine which among the genes is involved in the maintenance of cell wall integrity. We examined the effects of lytic enzymes (10 $\mathrm{mg} / \mathrm{mL}$ lysing enzymes) on the three $\Delta M o g l n$ mutants. Fewer protoplasts were generated by the $\Delta M o g \ln 2$ mutant compared to wild-type Guy11, $\Delta M o g \ln 1$, and $\Delta M o g \ln 3$ strains (Figure 10C,D), indicating either the cell wall structure was altered, making it less resistant to degradation by lytic enzymes, or the membrane and cell wall were breached as a result of excess rupture, thus leading to poor protoplast recovery. In rice blast fungus, reduced Mps1 phosphorylation was previously reported in a mutant with altered cell wall integrity [65]. Therefore, we performed western blot assay to determine the phosphorylation of Mps1 in the three MoGLN mutants. Mps1 phosphorylation remained unchanged in the $\Delta M \log \ln 1$ and $\Delta M \log \ln 3$ strains, and it was remarkably reduced in the $\Delta M o g \ln 2$ mutant, indicating cell wall defects associated with loss of MoGLN2 (Figure 10E). Taken together, these results indicate MoGLN2 is important in maintaining cell wall integrity in rice blast fungus.

\subsection{Intracellular Levels of Other Amino Acids Were Higher in $\Delta$ Mogln2 Mutant}

In Saccharomyces cerevisiae, reduced glutamine levels detected in a hypo-osmorality GLN1 mutant resulted in increased intracellular amounts of the other amino acids, except for proline [66]. To confirm whether low levels of glutamine affected the concentration of other amino acids in the $\Delta M o g \ln 2$ mutant, the Guy11 and $\Delta M o g \ln 2$ strains were cultured in liquid CM medium for three days and then transferred to MM medium for an additional three days. Mycelial samples were then used to detect the concentration of other amino acids. The steady-state intracellular pools of almost all the amino acids detected were found to have increased in $\Delta$ Mogln2 (Table 1). Analysis of RNA sequencing data for the Guy11 and $\Delta$ Mogln 2 mutants showed that increased intracellular amino acid levels in $\Delta M o g \ln 2$ correlated with the expression of genes related to amino acid biosynthesis, as RNA sequencing transcriptome data showed that the majority of genes, including those involved in translation, amino acid activation, tRNA aminoacylation, tRNA aminoacylation for protein translation, amide biosynthetic process, and peptide biosynthetic process, were found to be differentially up-regulated in the $\Delta M o g \ln 2$ mutant (Figure 13A). This confirms that reduced glutamine levels in $\Delta M o g \ln 2$ resulted in increased biosynthesis of other amino acids.

\subsection{2. $\Delta$ Mogln2 Is Hypersensitive to Oxidative Stress}

In rice fungal pathogens, sensitivity to oxidative stress using hydrogen peroxide is well documented [67-69]. To investigate the contributions of the three MoGLN genes in oxidative stress tolerance in rice blast fungus, we observed the mycelia growth of the three respective mutants on $\mathrm{CM}$ medium amended with $2.5 \mathrm{mM}$ and $5 \mathrm{mM}$ concentrations of hydrogen peroxide $\left(\mathrm{H}_{2} \mathrm{O}_{2}\right)$. We established that mycelial growth of the $\Delta M \operatorname{Mog} \ln 1$ mutant was moderately inhibited on $\mathrm{CM}$ medium containing $5 \mathrm{mM} \mathrm{H}_{2} \mathrm{O}_{2}$, while the $\Delta M \log \ln 3$ mutant exhibited less sensitivity both on $2.5 \mathrm{mM}$ and $5 \mathrm{mM}$ concentrations of $\mathrm{H}_{2} \mathrm{O}_{2}$ relative to the WT strain (Figure $11 \mathrm{~A}, \mathrm{~B}$ ). However, the $\Delta M \operatorname{Mog} \ln 2$ mutant was hypersensitive to both $2.5 \mathrm{mM}$ and $5 \mathrm{mM} \mathrm{H}_{2} \mathrm{O}_{2}$ concentrations (Figure 11A,B), suggesting therefore that MoGLN2 could be involved in the regulation of oxidative stress tolerance in rice blast fungus. 
A

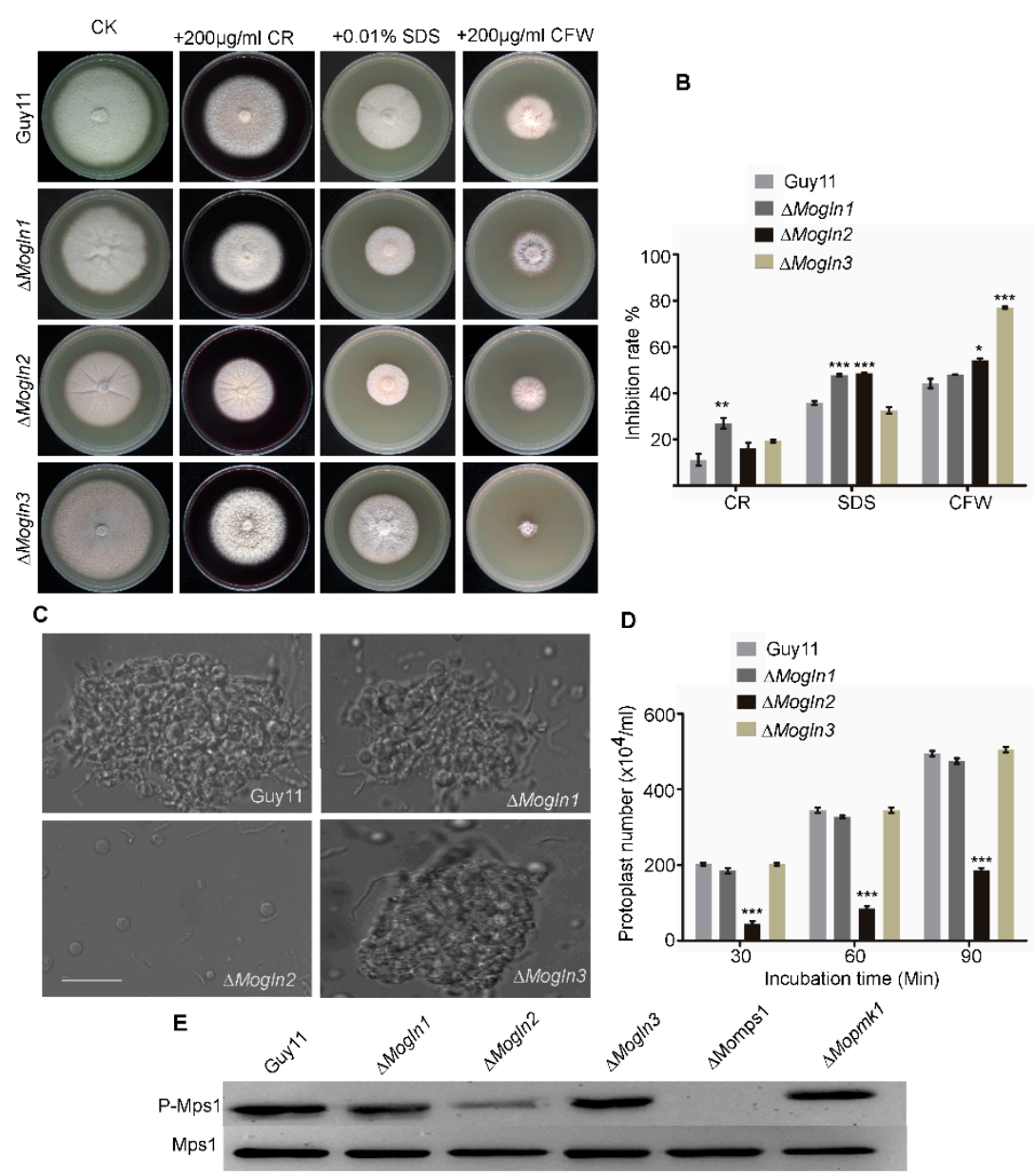

Figure 10. MoGLN2 is essential for maintenance of cell wall integrity in M. oryzae. (A) The Guy11 and MoGLN mutants were cultured on CM medium supplemented with $(200 \mu \mathrm{g} / \mathrm{mL} \mathrm{CR}, 0.01 \%$ SDS, and $200 \mu \mathrm{g} / \mathrm{mL} \mathrm{CFW}$ ) at $28^{\circ} \mathrm{C}$ for 8 days before being photographed. (B) Graph showing inhibition rate of WT and mutant strains. Inhibition rate was compared to the growth rate of each untreated control (Inhibition rate $=($ the diameter of untreated strain - the diameter of treated strain $) /($ the diameter of untreated strain $\times 100 \%)$ ). Three independent repeats were performed, with similar results obtained. (C) Light microscopic examination of protoplast release after treatment with cell-wall-degrading enzymes for $30 \mathrm{~min}, 60 \mathrm{~min}$, and $90 \mathrm{~min}$ at $28{ }^{\circ} \mathrm{C}$. Bar $=10 \mu \mathrm{m}$ (D) Graphical representation of protoplast release assay for the WT and three MoGLN mutants. (E) Phosphorylation of MoMps1 in WT and three MoGLN mutants, $\triangle M o p s 1$, and $\triangle M o p m k 1$. Proteins were prepared from mycelia inoculated in liquid CM, and the phosphorylated MoMps1 was detected by binding of the antiphospho-p44/42 antibody, with the Mpk1 antibody as a control. The phosphorylation level of MoMps1 in the $\Delta M o g \ln 2$ strains indicated the reduced activation of MoMps1. Statistical results for growth inhibition rate and protoplast results were obtained from at least three independent replicates. Error bars represent standard deviations. Asterisks indicate statistically significant differences ${ }^{*}, p<0.005^{* *}, p<0.01$, $* * *, p<0.001$; one-way ANOVA was used to analyze data with Tukey's multiple-comparison test in GraphPad Prism 8). 
Table 1. Intracellular free amino acids.

\begin{tabular}{|c|c|c|c|c|c|}
\hline \multirow{3}{*}{ Amino Acid } & \multirow{3}{*}{$p$-Value } & \multicolumn{2}{|c|}{ Guy11 } & \multicolumn{2}{|c|}{$\Delta M o g \ln 2$} \\
\hline & & \multicolumn{2}{|c|}{ Mean } & \multicolumn{2}{|c|}{ Mean } \\
\hline & & \multicolumn{2}{|c|}{$(\mu g / g) S D$} & \multicolumn{2}{|c|}{$(\mu g / g) S D$} \\
\hline Alanine & 0.004 & 2158.11 & 41.42232 & 3521.255 & 111.47538 \\
\hline Serine & 0.015 & 394.285 & 88.46613 & 911.845 & 13.73908 \\
\hline Proline & 0.01 & 234.075 & 22.90319 & 1054.775 & 116.4817 \\
\hline Valine & 0.008 & 399.47 & 1.24451 & 1125.905 & 90.12076 \\
\hline Isoleucine & 0.014 & 150.165 & 24.71338 & 604.355 & 73.88559 \\
\hline Threonine & 0.03 & 412.59 & 9.48937 & 2922.265 & 405.98536 \\
\hline Aspartate & 0.097 & 287.76 & 51.36424 & 503.8 & 89.35001 \\
\hline Leucine & 0 & 219.82 & 3.73352 & 880.38 & 7.43876 \\
\hline Asparagine & 0.012 & 119.015 & 35.65939 & 421.79 & 31.09856 \\
\hline Lysine & 0.038 & 2444.65 & 479.2487 & $17,505.03$ & 4272.45938 \\
\hline Glutamate & 0.024 & 1731.585 & 84.53462 & 4348.05 & 583.39138 \\
\hline Methionine & 0.026 & 50.34 & 3.6911 & 186.5 & 31.72081 \\
\hline Histidine & 0.094 & 474.54 & 36.44428 & 1734.325 & 585.7319 \\
\hline Phenylalanine & 0.007 & 4.1295 & 4.1295 & 460.365 & 38.33226 \\
\hline Arginine & 0.282 & 2692.36 & 310.1512 & 4231.13 & 1456.97938 \\
\hline Tryptophan & 0.002 & 50.755 & 0.3182 & 241.79 & 12.37437 \\
\hline Tyrosine & 0.064 & 271.06 & 60.99503 & 1841.21 & 589.82605 \\
\hline
\end{tabular}

SD represents standard deviation; mean is the average of two independent replicates.

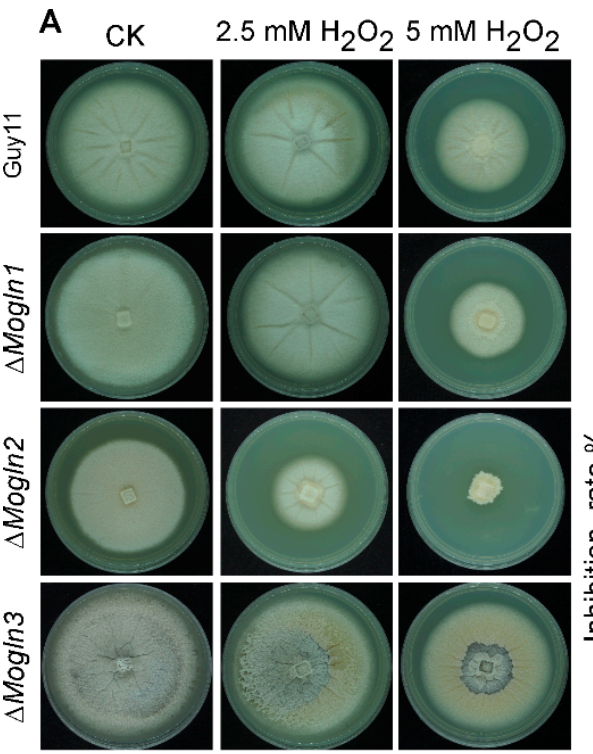

B

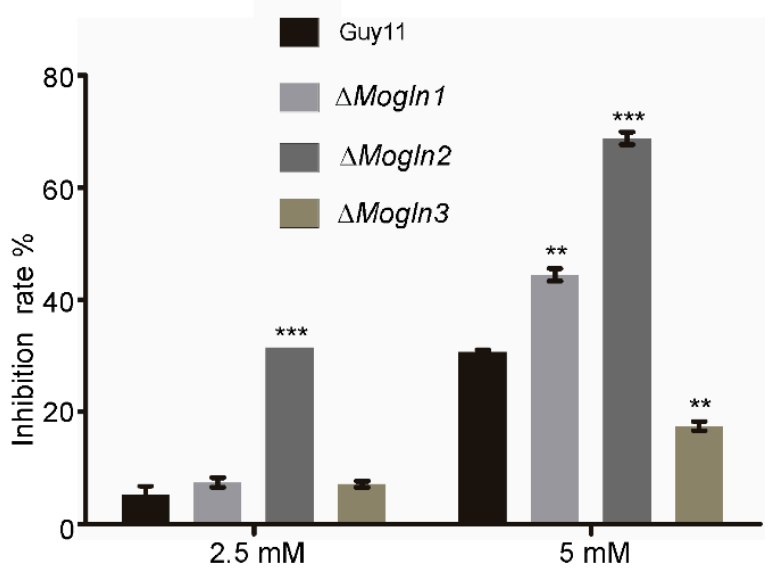

Figure 11. Sensitivity of the three MoGLN mutants to $\mathrm{H}_{2} \mathrm{O}_{2}$. (A) Growth phenotype of the WT and MoGLN mutants under oxidative stress. The WT and three mutant strains were inoculated on $\mathrm{CM}$ agar medium with or without $2.5 \mathrm{mM} \mathrm{H}_{2} \mathrm{O}_{2}$ and $5 \mathrm{mM} \mathrm{H}_{2} \mathrm{O}_{2}$ and cultured at $28^{\circ} \mathrm{C}$ for 10 days. (B) The colony diameters of the strain tested were measured, and statistical analysis was performed. The growth inhibition rate was compared to the growth rate of each untreated control (Inhibition rate $=($ the diameter of untreated strain - the diameter of treated strain $) /($ the diameter of untreated strain $\times 100 \%)$ ). Three independent repeats were performed, with similar results obtained. Error bars denote the standard deviations from means obtained from three independent replicates. Asterisks indicate statistically significant differences $\left({ }^{* *}, p<0.01 ;{ }^{* * *}, p<0.001\right.$; one-way ANOVA was used to analyze data with Turkey's multiple-comparison test in Graph Pad Prism 8). 


\subsection{MoGLN2 Is Important for Melanin Biosynthesis in Rice Blast Fungus}

In fungal pathogens, mycelia and appressoria undergo melanization; appressorial melanization is important for the normal functioning of the appressorium. After being cultured in liquid CM medium for five days, we observed a darkening of mycelial color for Guy11, $\Delta M o g \ln 1$, and $\Delta M o g \ln 3$ strains, implying that Guy11, $\Delta M o g \ln 1$, and $\Delta M o g \ln 3$ could be undergoing hyphal melanization. In contrast, no clearly visible darkening of mycelia was observed in culture inoculated with $\Delta M o g \ln 2$ strains (Figure 12A). This prompted us to speculate that the failure of $\Delta M o g \ln 2$ mycelia to form black pigmentation could be a result of the repression of genes important for melanization. We then performed qPCR analysis to confirm the expression of these genes in the three MoGLN mutants. As expected, BUF1, RSY1, and ALB1 were found to be down-regulated in the $\triangle M o g \ln 2$ mutant (Figure 12B), indicating that the melanization defect exhibited by the $\Delta M o g \ln 2$ strain is a result of reduced expression of these genes. Based on these results, we conclude that MoGLN2 plays a crucial role in the regulation of hyphal melanization in rice blast fungus.

A
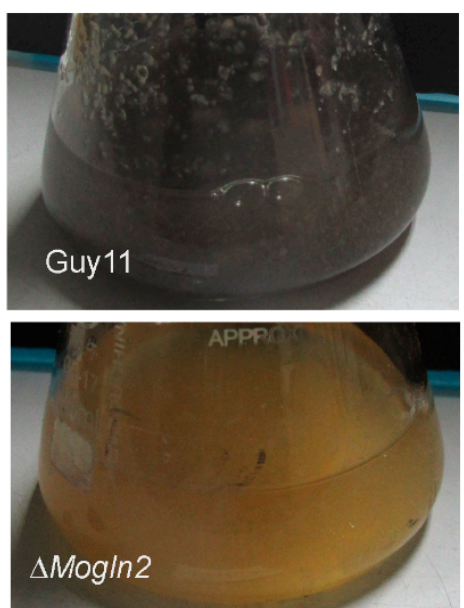
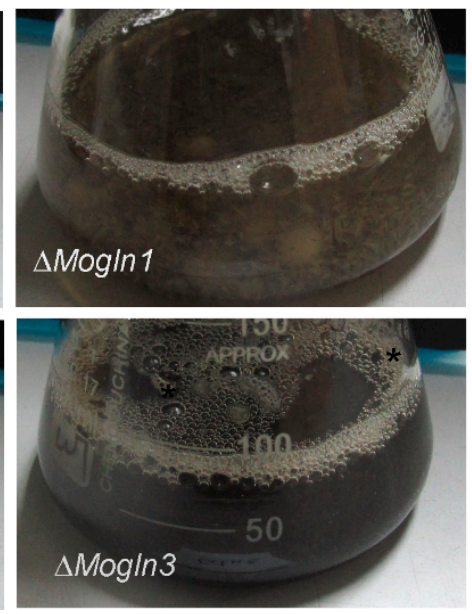

B

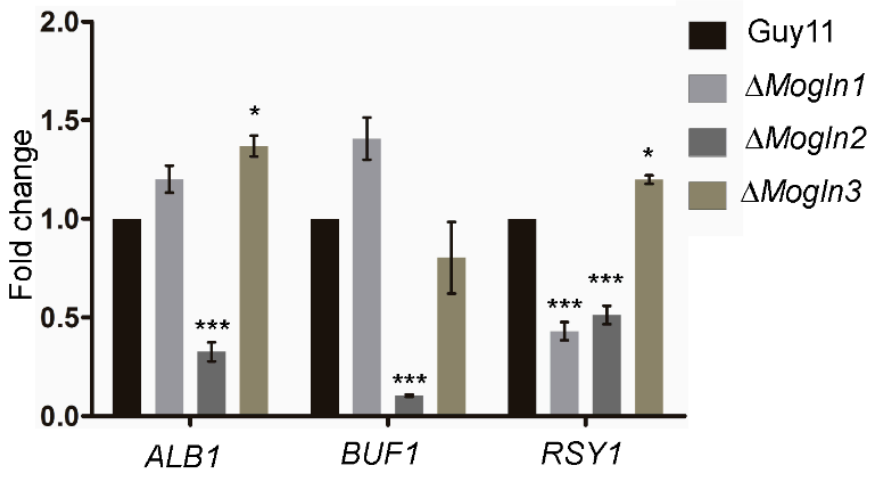

Figure 12. MoGLN2 is required for hyphal melanization in M. oryzae. (A) Mycelial growth in liquid CM medium showing impaired hyphal melanization as a result of MoGLN2 gene. (B) qRT-PCR analysis of the expression levels of genes important for melanin biosynthesis in mycelium grown in liquid CM. Error bars denote the standard deviations from means obtained from three independent replicates. Asterisks indicate statistically significant differences $\left({ }^{*}, p<0.005 ;{ }^{* *}, p<0.001\right.$; one-way ANOVA was used to analyze data with Tukey's multiple-comparison test in GraphPad Prism 8).

\subsection{Differentially Expressed in $\Delta$ Mogin2 Mutant}

Considering the dramatic phenotype exhibited by $\Delta M o g \ln 2$, we performed RNA sequencing and analyzed the transcriptome data for Guy11 and $\Delta M o g \ln 2$. This was aimed at establishing which genes were differentially expressed after deletion of MoGLN2. We established that 3703 genes were differentially expressed, including 1819 and 1884 up-regulated and down-regulated genes, respectively (Figure S1A,B). Gene Ontology (GO) and KEGG enrichment analysis for the genes up-regulated and down-regulated in $\Delta M o g \ln 2$ showed many enriched GO terms for up-regulated genes, including those that are involved as ribosome, translation, peptide biosynthetic process, purine nucleoside monophosphate metabolic process, ribonucleotide biosynthetic process, and obsolete cytosolic part (Figure 13A). KEGG analysis showed three enriched pathways, including Ribosome, oxidative phosphorylation' and aminoacyl-tRNA biosynthesis (Figure S2A). For down-regulated genes, we obtained fewer enriched results. In GO enrichment analysis, we discovered the term transmembrane transporter activity, peroxisome, and lipid catabolic process were enriched (Figure 13B). Moreover, we only discovered two enriched 
pathways, including microbial metabolism in diverse environments and $\mathrm{ABC}$ transporters (Figure S2B).

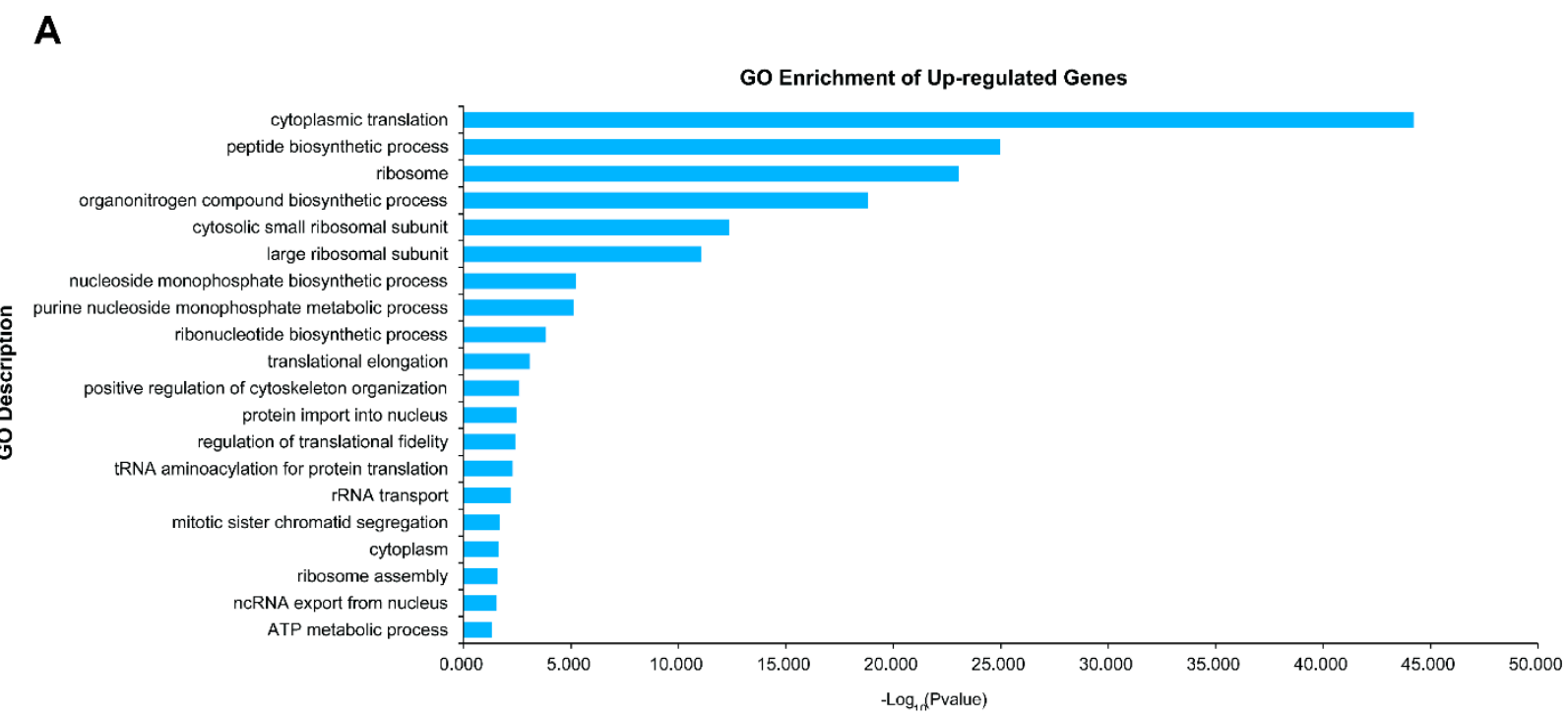

B

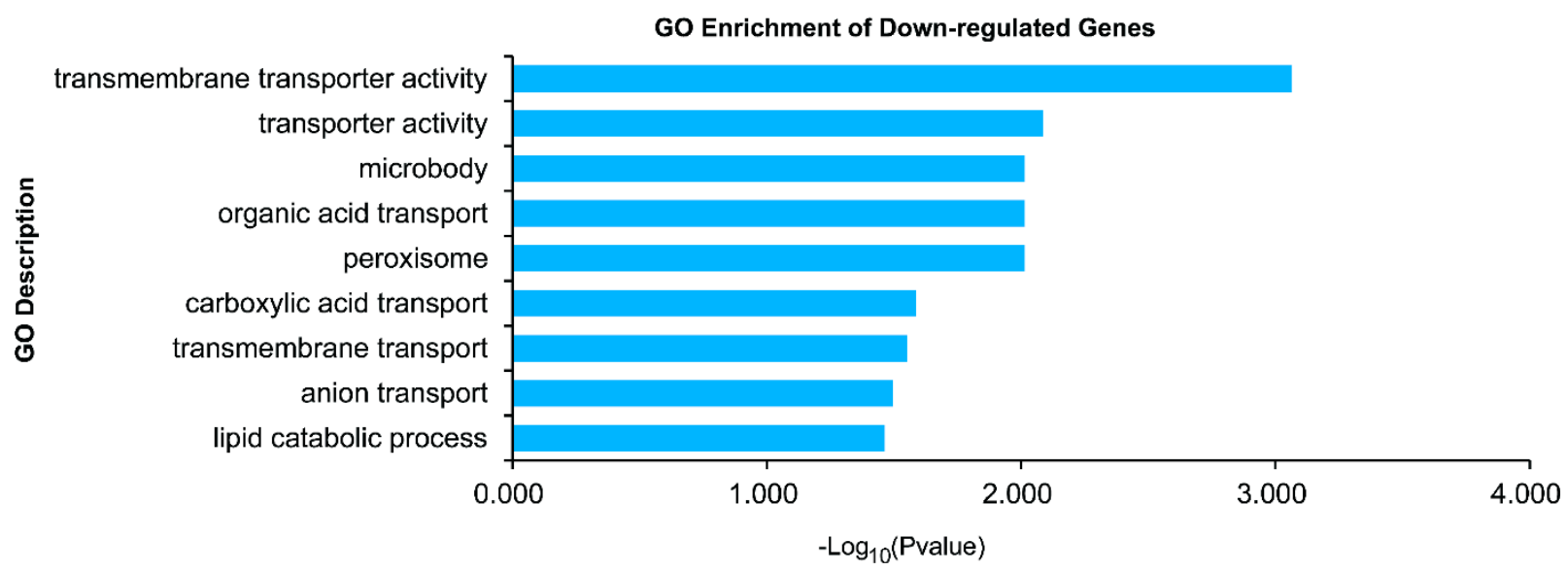

Figure 13. Molecular functions of the genes up-regulated (A) and down regulated (B) in $\Delta M o g \ln 2$ at a two-fold expression threshold based on the Gene Ontology (GO) terms.

\subsection{Subcelullar Localization of MoGLN Genes}

To evaluate the subcellular localization of MoGln1, MoGln2, and MoGln3 in rice blast fungus, the MoGLN1, MoGLN2, and MoGLN3 genes, with their corresponding native promoters, were fused in the C-terminus region of GFP and cloned in the pKNTG vector containing neomycin-resistant genes [70]. The constructs were separately transformed in their respective mutant protoplast. Results obtained showed that MoGln1-GFP, MoGln2GFP, and MoGln3-GFP were all targeted to the cytoplasm in growing hyphae, conidia, and appressorium (Figure $14 \mathrm{~A}-\mathrm{C}$ ), thus indicating the three MoGLN genes are all located in the cytoplasm in rice blast fungus. 
A

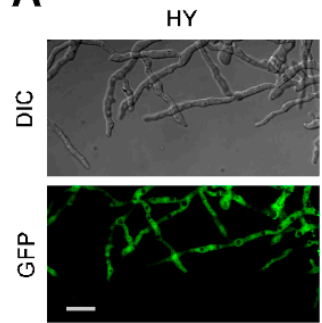

co
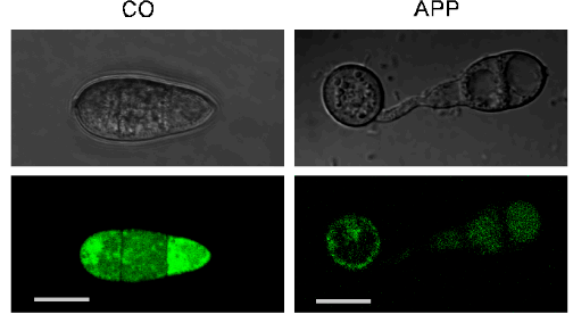

B

$\mathrm{HY}$

co

APP

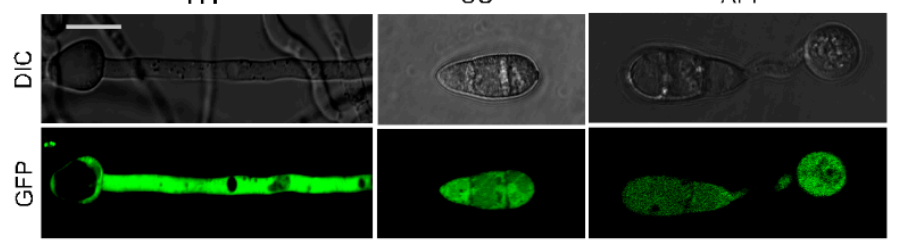

C

HY
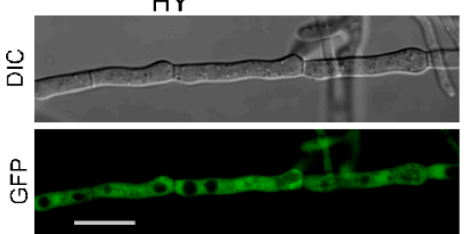

co

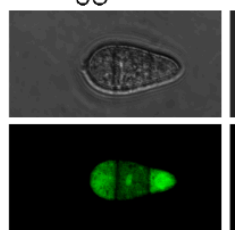

APP

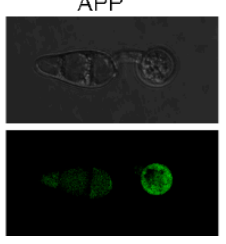

Figure 14. Subcellular localization of MoGln1, MoGln2, and MoGln3 in rice blast fungus. (A-C) The localization pattern of MoGln1-GFP, MoGln2-GFP, and MoGln3-GFP in hyphae, conidia, and appressorium. Localization of MoGln1-GFP, MoGln2-GFP, and MoGln3-GFP were examined by Nikon laser confocal, scale bar $=10 \mu \mathrm{m}$, MoGln1-GFP hyphae scale bar $=5 \mu \mathrm{m}$.

\section{Discussion}

Glutamine synthetase (GS) catalyzes the biosynthetic pathway involved in the synthesis of glutamine, and thus plays an important role in the assimilation of nitrogen. These enzymes have been extensively characterized in bacteria [41,71] and some filamentous fungi $[34,72]$. However, their functions in rice blast fungus remain uncharacterized. In this study, we identified and performed functional analysis of the three genes that encode glutamine synthetase (GLN1, GLN2, and GLN3) in M. oryzae. Upon deletion of each of the GS genes, we established that both MoGLN1 and MoGLN3 had no effect on the vegetative growth of $M$. oryzae. However, $\triangle M o g \ln 2$ was significantly reduced in growth on CM, SDC, and OTM. These findings echoed a previous study where glutamine synthetase GlnA1 of Mycobacterium tuberculosis was required for growth in human THP-1 macrophages and guinea pigs [41]. The $\Delta M o g \ln 2$ was attenuated in growth on MM medium, and supplementation of MM medium with L-glutamine rescued the growth defects, demonstrating that glutamine auxotroph in rice blast fungus occurs via inactivation of a single copy of GS (MoGLN2); this differs from a previous study with Sinorhizobium meliloti (formerly Rhizobium meliloti) that required all three GS genes to be inactivated to generate a strain that was auxotrophic for L-glutamine [73].

Intracellular glutamine test assays showed that glutamine levels were significantly reduced in $\Delta M o g \ln 2$, remained unchanged in $\Delta M o g \ln 1$, and were significantly higher in $\Delta M o g \ln 3$. An explanation for unchanged glutamine levels in the $\Delta M o g \ln 1$ mutant might be that glutamine was being synthesized by MoGLN2. The higher glutamine levels in $\triangle M o g \ln 3$ were attributed to up-regulation of both MoGLN1 and MoGLN2. MoGLN1 was previously reported to reduce higher glutamine turnovers in the $\triangle a s d 4$ mutant after the MoGLN1 gene was deleted in the $\triangle a s d 4$ mutant background [57]. This shows that MoGLN1 might be involved in glutamine biosynthesis. Therefore, the likely source of low intracellular glutamine detected in $\triangle M o g \ln 2$ was from up-regulation of MoGLN1; however, MoGLN1 cannot supplement MoGLN2 to produce sufficient glutamine levels required for fungal development and pathogenicity. The amount of other amino acids was found to have 
significantly increased in $\Delta M o g \ln 2$ and correlates with a study on S. cerevisiae where the intracellular amount of other amino acids was found to have increased in the $S$. cerevisiae $\Delta S c g \ln 1$ mutant [66]. The increased levels of the other amino acids in $\Delta M o g \ln 2$ might be attributed to enhanced biosynthesis of amino acids, as our RNA sequencing data revealed that genes related to amino acid biosynthesis, such as those involved in translation, amino acid activation, tRNA aminoacylation, tRNA aminoacylation for protein translation, amide biosynthetic process, and peptide biosynthetic process, were found to be up-regulated in $\Delta M o g \ln 2$ mutants.

Like in many fungal pathogens, conidiation and appressorium development are key steps in the disease cycle of M. oryzae. Upon landing on the host surface, the conidia begin to produce germ tubes and eventually develop into a specialized infection structure called appressorium, with $8 \mathrm{MPa}$ turgor pressure at the tips, which helps the fungus penetrate host cell barriers [39]. Analysis of the conidiation profiles of the three MoGLN mutants showed that both $\Delta M o g \ln 1$ and $\Delta M o g \ln 3$ had no effect on the asexual process in rice blast fungus. However, $\Delta M o g \ln 2$ failed to produce conidia in the different sporulation media tested. This observation was consistent with stage-specific qRT-PCR results, which showed that MoGLN2 was highly expressed at the conidiation stage. It is well shown that the aerial hyphal formation plays a crucial role during conidiophores differentiation and conidiation [74-77]. The $\Delta M o g \ln 2$ exhibited hyphal autolysis, with poor development of aerial hyphal on sporulation media SDC, OTM, and rice bran, indicating that the conidiation defects of the mutant may be due to the inability to form conidiophores. Sporulation defects of the mutant catalyzing amino acid biosynthetic process could be remediated by adding corresponding exogenous amino acid [78,79]. In this study, several attempts were made to supplement different sporulation media with L-glutamine concentration of $1 \mathrm{mM}, 2 \mathrm{mM}$, $5 \mathrm{mM}, 10 \mathrm{mM}, 20 \mathrm{mM}, 40 \mathrm{mM}$, and $60 \mathrm{mM}$; however, conidiogenesis could still not be restored by $\Delta M o g \ln 2$. L-glutamine was reported to be unstable compared to the other amino acids in aqueous solution [80] and given the long incubation time required for activation of conidiation in M. oryzae on sporulation medium (at least 10 days in dark and three days in light), it is most likely that a substantial amount of the initial L-glutamine is degraded in the culture medium. This could be a possible reason why exogenous L-glutamine did not rescue conidiation defects in $\Delta M o g \ln 2$.

Glutamine has been reported as one of the amino acids required by fungal pathogens during host colonization for successful infection to occur. For instance, analysis of amino acid changes during sunflower infection by Botrytis cinerea showed glutamine derived from the host was required by the fungus during in planta infection [81]. Moreover, colonization of Piriformospora indica to its host during in planta infection required glutamine [82]. In this study both $\Delta M o g \ln 1$ and $\Delta M o g \ln 3$ had a sufficient amount of glutamine, and thus they were able to colonize the barley cells and cause infection. However, the $\Delta M o g \ln 2$ mutant, which had low levels of intracellular glutamine, failed to form appressorium-like structures and thus was completely nonpathogenic on barley leaves. The failure of the $\Delta M \log \ln 2$ mutant to cause infection on barley leaves echoed previous studies with Mycobacterium species, where loss of function of GLN1A and GLN1 resulted in attenuated virulence on their respective hosts [41,83]. Our findings and previous studies on Mycobacterium species clearly demonstrate that glutamine biosynthesis mediated by glutamine synthetase is a critical process for pathogenic microorganisms to cause infection in their host.

The fungal cell wall is a vital structure with great plasticity and is crucial for maintaining cellular integrity and viability. The cell wall plays an important role in different functions, including controlling cellular permeability and cushioning the cell from osmotic and mechanical stress [84-86]. In addition, the cell wall facilitates smooth interactions of the cellular components with the external environment through adhesins and a large number of receptors; upon their activation, triggers signal transductions inside the cell [84]. Variations in nutrient availability result in changes in the expression of enzymes required for cell wall biosynthetic enzymes. It was previously reported that the loss of function of glutamine synthetase in $S$. cerevisiae resulted in $\Delta S c g \ln 1$ mutant showing a defect in cell wall 
integrity [66]. Furthermore, glutamine synthetase GLN1- $A$ in pathogenic Mycobacterium bovis was reported to be essential in cell wall resistance [83]. Similarly in this study, we established that the $\Delta M o g \ln 2$ mutants displayed increased sensitivity to cell wall stressors when cultured on $\mathrm{CM}$ medium containing (CR, CFW, and SDC). Moreover, less protoplast was formed in the $\Delta M o g \ln 2$ mutant compared to Guy11, $\Delta M o g \ln 1$, and $\Delta M o g \ln 3$ after being treated with lytic enzyme, implying an altered cell wall structure and making it resistant to degradation by lytic enzymes. Mps1 phosphorylation has been used as a marker for cell wall integrity tests [65]. In this study, the phosphorylation level of MoMps1 was decreased in $\Delta M o g \ln 2$ but not $\Delta M o g \ln 1$ and $\Delta M o g \ln 3$, further indicating the involvement of MoGLN2 in cell wall fungal cell integrity. Our findings on the contribution of MoGLN2 in the maintenance of cell wall integrity in rice blast fungus and previous studies on GLN1 of S. cerevisiae and GLN1-A in pathogenic Mycobacterium bovis demonstrate the important role of glutamine synthetase in cell wall integrity in living organisms.

It has been well demonstrated that ROS homeostasis is essential for fungal development $[87,88]$. In rice blast fungus, loss of MoSEC22 resulted in reduced intracellular ROS levels, with the mutant being highly sensitive to $\mathrm{H}_{2} \mathrm{O}_{2}$ and losing its virulence [89]. In this study, deletion of $\Delta M o g \ln 2$ was highly sensitive both on $2 \mathrm{mM}$ and $5 \mathrm{mM} \mathrm{H}_{2} \mathrm{O}_{2}$, indicating that MoGLN2 likely plays an important role in oxidative stress tolerance in rice blast fungus.

In summary, we established that among the three MoGLN genes, MoGLN2 is required for glutamine biosynthesis and is essential for growth, conidiogenesis, appressorium formation, and pathogenicity. Moreover, we confirmed that MoGLN2 is involved in the maintenance of cell wall integrity and oxidative stress tolerance in rice blast fungus. These findings provide an attractive target for the development of antifungal agents required to control the devastating effects of plant fungal pathogens.

Supplementary Materials: The following are available online at https://www.mdpi.com/article/ 10.3390/jof7060463/s1, Figure S1, Heatmap representation of differentially expressed genes upon deletion of MoGLN2; Figure S2, KEGG Enrichment for up-regulated and down-regulated pathways in $\Delta M o g \ln 2$ mutant: (A) KEGG enrichment for up-regulated genes (B) KEGG enrichment for downregulated genes; Table S1, Primers used in this study.

Author Contributions: Z.W., W.T. and O.A. designed this study; O.A., M.W., L.L., W.B., B.L. and A.S. performed experiments, analyzed all data, and wrote the initial manuscript; all authors contributed to writing and editing of the final manuscript. All authors have read and agreed to the published version of the manuscript.

Funding: The research was supported by funds from the Natural Science Foundation of China (31601584) and the Science Fund for Distinguished Young Scholars of Fujian Agriculture and Forestry University to W.T. (KXJQ17020), and the Special Fund Project for Science and Technology Innovation of Fujian Agriculture and Forestry University to W.T. (CXZX2020014A).

Institutional Review Board Statement: Not applicable.

Informed Consent Statement: Not applicable.

Data Availability Statement: Not applicable.

Acknowledgments: We are grateful to Justice Norvienyeku and Stefan Olsson for advice and discussion on the project.

Conflicts of Interest: The authors declare no conflict of interest.

\section{References}

1. Cooper, A.J.; Jeitner, T.M. Central role of glutamate metabolism in the maintenance of nitrogen homeostasis in normal and hyperammonemic brain. Biomolecules 2016, 6, 16. [CrossRef]

2. Curi, R.; Lagranha, C.J.; Doi, S.Q.; Sellitti, D.; Procópio, J.; Pithon-Curi, T.C.; Corless, M.; Newsholme, P. Molecular mechanisms of glutamine action. J. Cell. Physiol. 2005, 204, 392-401. [CrossRef]

3. Albrecht, J.; Sidoryk-Wegrzynowicz, M.; Zielinska, M.; Aschner, M. Roles of glutamine in neurotransmission. Neuron Glia Biol. 2010, 6, 263. [CrossRef] 
4. Carr, E.L.; Kelman, A.; Wu, G.S.; Gopaul, R.; Senkevitch, E.; Aghvanyan, A.; Turay, A.M.; Frauwirth, K.A. Glutamine uptake and metabolism are coordinately regulated by ERK/MAPK during T lymphocyte activation. J. Immunol. 2010, 185, 1037-1044. [CrossRef] [PubMed]

5. Haeussinger, D.; Schliess, F. Glutamine metabolism and signaling in the liver. Front. Biosci. 2007, 12, 371-391. [CrossRef] [PubMed]

6. Watford, M. Glutamine metabolism and function in relation to proline synthesis and the safety of glutamine and proline supplementation. J. Nutr. 2008, 138, 2003S-2007S. [CrossRef]

7. Lane, A.N.; Fan, T.W.-M. Regulation of mammalian nucleotide metabolism and biosynthesis. Nucleic Acids Res. 2015, 43, 2466-2485. [CrossRef]

8. Caddick, M.X.; Peters, D.; Platt, A. Nitrogen regulation in fungi. Antonie Van Leeuwenhoek 1994, 65, 169-177. [CrossRef]

9. Wiemann, P.; Tudzynski, B. The nitrogen regulation network and its impact on secondary metabolism and pathogenicity. In Fusarium: Genomics, Molecular and Cellular Biology; Caister Academic Press: Norfolk, UK, 2013; pp. 111-142.

10. Crespo, J.L.; Powers, T.; Fowler, B.; Hall, M.N. The TOR-controlled transcription activators GLN3, RTG1, and RTG3 are regulated in response to intracellular levels of glutamine. Proc. Natl. Acad. Sci. USA 2002, 99, 6784-6789. [CrossRef]

11. Camargo, A.; Llamas, Á.; Schnell, R.A.; Higuera, J.J.; González-Ballester, D.; Lefebvre, P.A.; Fernández, E.; Galván, A. Nitrate signaling by the regulatory gene NIT2 in Chlamydomonas. Plant Cell 2007, 19, 3491-3503. [CrossRef] [PubMed]

12. Magasanik, B.; Kaiser, C.A. Nitrogen regulation in Saccharomyces cerevisiae. Gene 2002, 290, 1-18. [CrossRef]

13. Wiame, J.-M.; Grenson, M.; Ars, H.N., Jr. Nitrogen catabolite repression in yeasts and filamentous fungi. In Advances in Microbial Physiology; Elsevier: Amsterdam, The Netherlands, 1985; Volume 26, pp. 1-88.

14. Rutherford, J.C.; Chua, G.; Hughes, T.; Cardenas, M.E.; Heitman, J. A Mep2-dependent transcriptional profile links permease function to gene expression during pseudohyphal growth in Saccharomyces cerevisiae. Mol. Biol. Cell 2008, 19, 3028-3039. [CrossRef]

15. Wedler, F.C.; Horn, B.R. Catalytic mechanisms of glutamine synthetase enzymes. Studies with analogs of possible intermediates and transition states. J. Biol. Chem. 1976, 251, 7530-7538. [CrossRef]

16. Liaw, S.H.; Eisenberg, D. Structural model for the reaction mechanism of glutamine synthetase, based on five crystal structures of enzyme-substrate complexes. Biochemistry 1994, 33, 675-681. [CrossRef] [PubMed]

17. Hirel, B.; McNally, S.F.; Gadal, P.; Sumar, N.; Stewart, G.R. Cytosolic glutamine synthetase in higher plants: A comparative immunological study. Eur. J. Biochem. 1984, 138, 63-66. [CrossRef] [PubMed]

18. Hennig, S.B.; Anderson, W.B.; Ginsburg, A. Adenosine triphosphate: Glutamine synthetase adenylyltransferase of Escherichia coli: Two active molecular forms. Proc. Natl. Acad. Sci. USA 1970, 67, 1761-1768. [CrossRef]

19. Jiang, P.; Mayo, A.E.; Ninfa, A.J. Escherichia coli glutamine synthetase adenylyltransferase (ATase, EC 2.7. 7.49): Kinetic characterization of regulation by PII, PII-UMP, glutamine, and $\alpha$-ketoglutarate. Biochemistry 2007, 46, 4133-4146. [CrossRef]

20. Al-Gharawi, A.; Moore, D. Factors affecting the amount and the activity of the glutamate dehydrogenases of Coprinus cinereus. Biochim. Biophys. Acta (BBA) Gen. Subj. 1977, 496, 95-102. [CrossRef]

21. Brown, J.; Masuchi, Y.; Robb, F.; Doolittlel, W. Evolutionary relationships of bacterial and archaeal glutamine synthetase genes. J. Mol. Evol. 1994, 38, 566-576. [CrossRef] [PubMed]

22. Eisenberg, D.; Gill, H.S.; Pfluegl, G.M.; Rotstein, S.H. Structure-function relationships of glutamine synthetases. Biochim. Biophys. Acta (BBA) Protein Struct. Mol. Enzymol. 2000, 1477, 122-145. [CrossRef]

23. Almassy, R.J.; Janson, C.A.; Hamlin, R.; Xuong, N.; Eisenberg, D. Novel subunit-Subunit interactions in the structure of glutamine synthetase. Nature 1986, 323, 304-309. [CrossRef]

24. Wyatt, K.; White, H.E.; Wang, L.; Bateman, O.A.; Slingsby, C.; Orlova, E.V.; Wistow, G. Lengsin is a survivor of an ancient family of class I glutamine synthetases re-engineered by evolution for a role in the vertebrate lens. Structure 2006, 14, 1823-1834. [CrossRef]

25. Mathis, R.; Gamas, P.; Meyer, Y.; Cullimore, J.V. The presence of GSI-like genes in higher plants: Support for the paralogous evolution of GSI and GSII genes. J. Mol. Evol. 2000, 50, 116-122. [CrossRef] [PubMed]

26. Dávila, G.; Lara, M.; Guzmán, J.; Mora, J. Relation between structure and function of Neurospora crassa glutamine synthetase. Biochem. Biophys. Res. Commun. 1980, 92, 134-140. [CrossRef]

27. Mora, J. Glutamine metabolism and cycling in Neurospora crassa. Microbiol. Mol. Biol. Rev. 1990, 54, 293-304. [CrossRef]

28. Liaw, S.-H.; Jun, G.; Eisenberg, D. Interactions of nucleotides with fully unadenylylated glutamine synthetase from Salmonella typhimurium. Biochemistry 1994, 33, 11184-11188. [CrossRef] [PubMed]

29. Gunka, K.; Commichau, F.M. Control of glutamate homeostasis in Bacillus subtilis: A complex interplay between ammonium assimilation, glutamate biosynthesis and degradation. Mol. Microbiol. 2012, 85, 213-224. [CrossRef]

30. Wray, L.V., Jr.; Zalieckas, J.M.; Fisher, S.H. Bacillus subtilis glutamine synthetase controls gene expression through a protein-protein interaction with transcription factor TnrA. Cell 2001, 107, 427-435. [CrossRef]

31. Caggese, C.; Caizzi, R.; Barsanti, P.; Bozzetti, M.P. Mutations in the glutamine synthetase I (gsI) gene produce embryo-lethal female sterility in Drosophila melanogaster. Dev. Genet. 1992, 13, 359-366. [CrossRef]

32. Häberle, J.; Görg, B.; Rutsch, F.; Schmidt, E.; Toutain, A.; Benoist, J.-F.; Gelot, A.; Suc, A.-L.; Höhne, W.; Schliess, F. Congenital glutamine deficiency with glutamine synthetase mutations. N. Engl. J. Med. 2005, 353, 1926-1933. [CrossRef]

33. Bao, A.; Zhao, Z.; Ding, G.; Shi, L.; Xu, F.; Cai, H. The stable level of glutamine synthetase 2 plays an important role in rice growth and in carbon-nitrogen metabolic balance. Int. J. Mol. Sci. 2015, 16, 12713-12736. [CrossRef] 
34. Margelis, S.; D'Souza, C.; Small, A.J.; Hynes, M.J.; Adams, T.H.; Davis, M.A. Role of glutamine synthetase in nitrogen metabolite repression in Aspergillus nidulans. J. Bacteriol. 2001, 183, 5826-5833. [CrossRef]

35. Sasaki, Y.; Kojima, A.; Shibata, Y.; Mitsuzawa, H. Filamentous invasive growth of mutants of the genes encoding ammoniametabolizing enzymes in the fission yeast Schizosaccharomyces pombe. PLoS ONE 2017, 12, e0186028. [CrossRef]

36. Teichert, S.; Schönig, B.; Richter, S.; Tudzynski, B. Deletion of the Gibberella fujikuroi glutamine synthetase gene has significant impact on transcriptional control of primary and secondary metabolism. Mol. Microbiol. 2004, 53, 1661-1675. [CrossRef]

37. Talbot, N.J. On the trail of a cereal killer: Exploring the biology of Magnaporthe grisea. Annu. Rev. Microbiol. 2003, 57, 177-202. [CrossRef]

38. Dagdas, Y.F.; Yoshino, K.; Dagdas, G.; Ryder, L.S.; Bielska, E.; Steinberg, G.; Talbot, N.J. Septin-mediated plant cell invasion by the rice blast fungus, Magnaporthe oryzae. Science 2012, 336, 1590-1595. [CrossRef] [PubMed]

39. Howard, R.J.; Ferrari, M.A.; Roach, D.H.; Money, N.P. Penetration of hard substrates by a fungus employing enormous turgor pressures. Proc. Natl. Acad. Sci. USA 1991, 88, 11281-11284. [CrossRef]

40. Kankanala, P.; Czymmek, K.; Valent, B. Roles for rice membrane dynamics and plasmodesmata during biotrophic invasion by the blast fungus. Plant Cell 2007, 19, 706-724. [CrossRef] [PubMed]

41. Tullius, M.V.; Harth, G.; Horwitz, M.A. Glutamine synthetase GlnA1 is essential for growth of Mycobacterium tuberculosis in human THP-1 macrophages and guinea pigs. Infect. Immun. 2003, 71, 3927-3936. [CrossRef]

42. Chen, J.; Zheng, W.; Zheng, S.; Zhang, D.; Sang, W.; Chen, X.; Li, G.; Lu, G.; Wang, Z. Rac1 is required for pathogenicity and Chm1-dependent conidiogenesis in rice fungal pathogen Magnaporthe grisea. PLoS Pathog. 2008, 4, e1000202. [CrossRef] [PubMed]

43. Goswami, R.S. Targeted gene replacement in fungi using a split-marker approach. In Plant Fungal Pathogens; Springer: New York, NY, USA, 2012; pp. 255-269.

44. Zhang, C.; Zong, H.; Zhuge, B.; Lu, X.; Fang, H.; Zhu, J.; Zhuge, J. Protoplast preparation and polyethylene glycol (PEG)-mediated transformation of Candida glycerinogenes. Biotechnol. Bioprocess Eng. 2016, 21, 95-102. [CrossRef]

45. Qi, Z.; Wang, Q.; Dou, X.; Wang, W.; Zhao, Q.; Lv, R.; Zhang, H.; Zheng, X.; Wang, P.; Zhang, Z. MoSwi6, an APSES family transcription factor, interacts with MoMps1 and is required for hyphal and conidial morphogenesis, appressorial function and pathogenicity of Magnaporthe oryzae. Mol. Plant Pathol. 2012, 13, 677-689. [CrossRef] [PubMed]

46. Wang, Y.; Pang, J.; Zheng, Y.; Jiang, P.; Gong, W.; Chen, X.; Chen, D. Genetic manipulation of the bifunctional gene, carRA, to enhance lycopene content in Blakeslea trispora. Biochem. Eng. J. 2017, 119, 27-33. [CrossRef]

47. Sambrook, J.; Fritsch, E.F.; Maniatis, T. Molecular Cloning: A Laboratory Manual; Cold Spring Harbor Laboratory Press: Cold Spring Harbor, NY, USA, 1989.

48. Lin, L.; Chen, X.; Shabbir, A.; Chen, S.; Chen, X.; Wang, Z.; Norvienyeku, J. A putative N-BAR-domain protein is crucially required for the development of hyphae tip appressorium-like structure and its plant infection in Magnaporthe Oryzae. Phytopathol. Res. 2019, 1, 1-15. [CrossRef]

49. Livak, K.J.; Schmittgen, T.D. Analysis of relative gene expression data using real-time quantitative PCR and the $2^{-\Delta \Delta C T}$ method. Methods 2001, 25, 402-408. [CrossRef] [PubMed]

50. Bruno, K.S.; Tenjo, F.; Li, L.; Hamer, J.E.; Xu, J.-R. Cellular localization and role of kinase activity of PMK1 in Magnaporthe grisea. Eukaryot. Cell 2004, 3, 1525-1532. [CrossRef]

51. Dong, Y.; Li, Y.; Zhao, M.; Jing, M.; Liu, X.; Liu, M.; Guo, X.; Zhang, X.; Chen, Y.; Liu, Y. Global genome and transcriptome analyses of Magnaporthe oryzae epidemic isolate 98-06 uncover novel effectors and pathogenicity-related genes, revealing gene gain and lose dynamics in genome evolution. PLoS Pathog. 2015, 11, e1004801. [CrossRef] [PubMed]

52. Langmead, B.; Salzberg, S.L. Fast gapped-read alignment with Bowtie 2. Nat. Methods 2012, 9, 357-359. [CrossRef]

53. Li, B.; Dewey, C.N. RSEM: Accurate transcript quantification from RNA-Seq data with or without a reference genome. BMC Bioinform. 2011, 12, 323. [CrossRef] [PubMed]

54. Love, M.I.; Huber, W.; Anders, S. Moderated estimation of fold change and dispersion for RNA-seq data with DESeq2. Genome Biol. 2014, 15, 550. [CrossRef]

55. McGinnis, S.; Madden, T.L. BLAST: At the core of a powerful and diverse set of sequence analysis tools. Nucleic Acids Res. 2004, 32, W20-W25. [CrossRef]

56. Liu, W.; Xie, Y.; Ma, J.; Luo, X.; Nie, P.; Zuo, Z.; Lahrmann, U.; Zhao, Q.; Zheng, Y.; Zhao, Y. IBS: An illustrator for the presentation and visualization of biological sequences. Bioinformatics 2015, 31, 3359-3361. [CrossRef]

57. Marroquin-Guzman, M.; Wilson, R.A. GATA-dependent glutaminolysis drives appressorium formation in Magnaporthe oryzae by suppressing TOR inhibition of cAMP/PKA signaling. PLoS Pathog. 2015, 11, e1004851. [CrossRef] [PubMed]

58. Kim, S.; Park, S.-Y.; Kim, K.S.; Rho, H.-S.; Chi, M.-H.; Choi, J.; Park, J.; Kong, S.; Park, J.; Goh, J. Homeobox transcription factors are required for conidiation and appressorium development in the rice blast fungus Magnaporthe oryzae. PLoS Genet. 2009, 5 , e1000757. [CrossRef] [PubMed]

59. de Jong, J.C.; McCormack, B.J.; Smirnoff, N.; Talbot, N.J. Glycerol generates turgor in rice blast. Nature 1997, 389, 244. [CrossRef]

60. Lussier, M.; White, A.-M.; Sheraton, J.; di Paolo, T.; Treadwell, J.; Southard, S.B.; Horenstein, C.I.; Chen-Weiner, J.; Ram, A.F.; Kapteyn, J.C. Large scale identification of genes involved in cell surface biosynthesis and architecture in Saccharomyces cerevisiae. Genetics 1997, 147, 435-450. [CrossRef] [PubMed]

61. Ram, A.F.; Wolters, A.; Hoopen, R.T.; Klis, F.M. A new approach for isolating cell wall mutants in Saccharomyces cerevisiae by screening for hypersensitivity to calcofluor white. Yeast 1994, 10, 1019-1030. [CrossRef] 
62. Bickle, M.; Delley, P.A.; Schmidt, A.; Hall, M.N. Cell wall integrity modulates RHO1 activity via the exchange factor ROM2. EMBO J. 1998, 17, 2235-2245. [CrossRef] [PubMed]

63. Shimizu, J.; Yoda, K.; Yamasaki, M. The hypo-osmolarity-sensitive phenotype of the Saccharomyces cerevisiae hpo2 mutant is due to a mutation in PKC1, which regulates expression of $\beta$-glucanase. Mol. Gen. Genet. MGG 1994, 242, 641-648. [CrossRef]

64. Wood, P.J.; Fulcher, R. Dye interactions. A basis for specific detection and histochemistry of polysaccharides. J. Histochem. Cytochem. 1983, 31, 823-826. [CrossRef]

65. Yin, Z.; Tang, W.; Wang, J.; Liu, X.; Yang, L.; Gao, C.; Zhang, J.; Zhang, H.; Zheng, X.; Wang, P. Phosphodiesterase MoPdeH targets MoMck1 of the conserved mitogen-activated protein (MAP) kinase signalling pathway to regulate cell wall integrity in rice blast fungus Magnaporthe oryzae. Mol. Plant Pathol. 2016, 17, 654-668. [CrossRef]

66. Shimizu, J.; Okumura, Y.; Yoda, K.; Yamasaki, M. A glutamine synthetase mutant of Saccharomyces cerevisiae shows defect in cell wall. J. Gen. Appl. Microbiol. 1997, 43, 157-162. [CrossRef] [PubMed]

67. Kim, K.-H.; Willger, S.D.; Park, S.-W.; Puttikamonkul, S.; Grahl, N.; Cho, Y.; Mukhopadhyay, B.; Cramer, R.A., Jr.; Lawrence, C.B. TmpL, a transmembrane protein required for intracellular redox homeostasis and virulence in a plant and an animal fungal pathogen. PLoS Pathog. 2009, 5, e1000653. [CrossRef]

68. Guo, M.; Guo, W.; Chen, Y.; Dong, S.; Zhang, X.; Zhang, H.; Song, W.; Wang, W.; Wang, Q.; Lv, R. The basic leucine zipper transcription factor Moatf1 mediates oxidative stress responses and is necessary for full virulence of the rice blast fungus Magnaporthe oryzae. Mol. Plant Microbe Interact. 2010, 23, 1053-1068. [CrossRef] [PubMed]

69. Fernandez, J.; Yang, K.T.; Cornwell, K.M.; Wright, J.D.; Wilson, R.A. Growth in rice cells requires de novo purine biosynthesis by the blast fungus Magnaporthe oryzae. Sci. Rep. 2013, 3, 2398. [CrossRef] [PubMed]

70. Yang, J.; Zhao, X.; Sun, J.; Kang, Z.; Ding, S.; Xu, J.-R.; Peng, Y.-L. A novel protein Com1 is required for normal conidium morphology and full virulence in Magnaporthe oryzae. Mol. Plant Microbe Interact. 2010, 23, 112-123. [CrossRef]

71. Harth, G.; Clemens, D.L.; Horwitz, M.A. Glutamine synthetase of Mycobacterium tuberculosis: Extracellular release and characterization of its enzymatic activity. Proc. Natl. Acad. Sci. USA 1994, 91, 9342-9346. [CrossRef]

72. Dunn-Coleman, N.S.; Garrett, R.H. The role of glutamine synthetase and glutamine metabolism in nitrogen metabolite repression, a regulatory phenomenon in the lower eukaryote Neurospora crassa. Mol. Gen. Genet. MGG 1980, 179, 25-32. [CrossRef]

73. Shatters, R.; Liu, Y.; Kahn, M. Isolation and characterization of a novel glutamine synthetase from Rhizobium meliloti. J. Biol. Chem. 1993, 268, 469-475. [CrossRef]

74. Adams, T.H.; Wieser, J.K.; Yu, J.-H. Asexual sporulation in Aspergillus nidulans. Microbiol. Mol. Biol. Rev. 1998, 62, 35-54. [CrossRef]

75. Garzia, A.; Etxebeste, O.; Herrero-Garcia, E.; Fischer, R.; Espeso, E.A.; Ugalde, U. Aspergillus nidulans FlbE is an upstream developmental activator of conidiation functionally associated with the putative transcription factor FlbB. Mol. Microbiol. 2009, 71, 172-184. [CrossRef]

76. Kikuma, T.; Arioka, M.; Kitamoto, K. Autophagy during conidiation and conidial germination in filamentous fungi. Autophagy 2007, 3, 128-129. [CrossRef]

77. Timberlake, W.E. Temporal and spatial controls of Aspergillus development. Curr. Opin. Genet. Dev. 1991, 1, 351-357. [CrossRef]

78. Yan, X.; Que, Y.; Wang, H.; Wang, C.; Li, Y.; Yue, X.; Ma, Z.; Talbot, N.J.; Wang, Z. The MET13 methylenetetrahydrofolate reductase gene is essential for infection-related morphogenesis in the rice blast fungus Magnaporthe oryzae. PLoS ONE 2013,8 , e76914.

79. Du, Y.; Zhang, H.; Hong, L.; Wang, J.; Zheng, X.; Zhang, Z. Acetolactate synthases MoIlv2 and MoIlv6 are required for infection-related morphogenesis in Magnaporthe oryzae. Mol. Plant Pathol. 2013, 14, 870-884. [CrossRef]

80. Khan, K.; Hardy, G.; McElroy, B.; Elia, M. The stability of L-glutamine in total parenteral nutrition solutions. Clin. Nutr. 1991, 10, 193-198. [CrossRef]

81. Dulermo, T.; Bligny, R.; Gout, E.; Cotton, P. Amino acid changes during sunflower infection by the necrotrophic fungus B. cinerea. Plant Signal. Behav. 2009, 4, 859-861. [CrossRef] [PubMed]

82. Lahrmann, U.; Ding, Y.; Banhara, A.; Rath, M.; Hajirezaei, M.R.; Döhlemann, S.; von Wirén, N.; Parniske, M.; Zuccaro, A. Host-related metabolic cues affect colonization strategies of a root endophyte. Proc. Natl. Acad. Sci. USA 2013, 110, 13965-13970. [CrossRef]

83. Chandra, H.; Basir, S.F.; Gupta, M.; Banerjee, N. Glutamine synthetase encoded by glnA-1 is necessary for cell wall resistance and pathogenicity of Mycobacterium bovis. Microbiology 2010, 156, 3669-3677. [CrossRef] [PubMed]

84. Ponton, J. The fungal cell wall and the mechanism of action of anidulafungin. Rev. Iberoam. Micol. 2008, 25, 78-82. [PubMed]

85. Gow, N.A.; Latge, J.-P.; Munro, C.A. The fungal cell wall: Structure, biosynthesis, and function. Microbiol. Spectr. 2017, 5, 267-292.

86. Agustinho, D.P.; Miller, L.C.; Li, L.X.; Doering, T.L. Peeling the onion: The outer layers of Cryptococcus neoformans. Mem. Inst. Oswaldo Cruz 2018, 113, e180040. [CrossRef] [PubMed]

87. Aguirre, J.; Ríos-Momberg, M.; Hewitt, D.; Hansberg, W. Reactive oxygen species and development in microbial eukaryotes. Trends Microbiol. 2005, 13, 111-118. [CrossRef] [PubMed]

88. Gessler, N.N.; Aver'yanov, A.A.; Belozerskaya, T. Reactive oxygen species in regulation of fungal development. Biochem. (Moscow) 2007, 72, 1091-1109. [CrossRef]

89. Song, W.; Dou, X.; Qi, Z.; Wang, Q.; Zhang, X.; Zhang, H.; Guo, M.; Dong, S.; Zhang, Z.; Wang, P. R-SNARE homolog MoSec22 is required for conidiogenesis, cell wall integrity, and pathogenesis of Magnaporthe oryzae. PLoS ONE 2010, 5, e13193. [CrossRef] 